\title{
RATE OF CONVERGENCE IN THE BREUER-MAJOR THEOREM VIA CHAOS EXPANSIONS
}

\author{
SEFIKA KUZGUN AND DAVID NUALART
}

\begin{abstract}
We show new estimates for the total variation and Wasserstein distances in the framework of the Breuer-Major theorem. The results are based on the combination of Stein's method for normal approximations and Malliavin calculus together with Wiener chaos expansions.
\end{abstract}

Mathematics Subject Classifications (2010): 60H15, 60H07, 60G15, 60F05.

\section{INTRODUCTION}

Suppose that $X=\left\{X_{n}, n \geq 0\right\}$ is a centered stationary Gaussian sequence of random variables with unit variance. For all $k \in \mathbb{Z}$, set $\rho(k)=\mathbb{E}\left(X_{0} X_{k}\right)$ if $k \geq 0$ and $\rho(k)=\rho(-k)$ if $k<0$. We say that a function $g \in L^{2}(\mathbb{R}, \gamma)$, where $\gamma$ is the standard Gaussian measure, has Hermite rank $d \geq 1$ if

$$
g(x)=\sum_{q=d}^{\infty} c_{q} H_{q}(x)
$$

where $c_{d} \neq 0$ and $H_{q}$ is the $q$-th Hermite polynomial. We will make use of the following condition that relates the covariance function $\rho$ to the Hermite rank of a function $g$ :

$$
\sum_{j \in \mathbb{Z}}|\rho(j)|^{d}<\infty .
$$

The Breuer-Major theorem (see 4]) says that, under condition (1.2), the sequence

$$
F_{n}:=\frac{1}{\sqrt{n}} \sum_{i=1}^{n} g\left(X_{i}\right)
$$

converges in law to the normal distribution $N\left(0, \sigma^{2}\right)$, where

$$
\sigma^{2}=\sum_{q=d}^{\infty} q ! c_{q}^{2} \sum_{k \in \mathbb{Z}} \rho(k)^{q} .
$$

The aim of this paper is to estimate the rate of convergence to zero of the total variation and Wasserstein distances between the normalized sequence

$$
Y_{n}:=\frac{F_{n}}{\sqrt{\operatorname{Var}\left(F_{n}\right)}}
$$

and the standard normal law $N(0,1)$, assuming minimal regularity and integrability conditions on the function $g$. To show these results we will apply a combination of Stein's method for normal approximations and techniques of Malliavin calculus, and we will make use of the

D. Nualart is supported by the NSF Grant DMS 1811181. 
Wiener chaos expansion of the random variable $F_{n}$. The combination of Stein's method with Malliavin calculus to study normal approximations was first developed by Nourdin and Peccati (see the pioneering work [9] and the monograph [10]). For random variables on a fixed Wiener chaos, these techniques provide a quantitative version of the Fourth Moment Theorem proved by Nualart and Peccati in [16].

Given a function $g \in L^{2}(\mathbb{R}, \gamma)$ with expansion (1.1), we denote by $A(g)$ the function in $L^{2}(\mathbb{R}, \gamma)$, whose Hermite coefficients are the absolute values of the coefficients of $g$, that is,

$$
A(g)(x)=\sum_{q=d}^{\infty}\left|c_{q}\right| H_{q}(x) .
$$

For any integer $k \geq 1$ and any real $p \geq 1$, we denote by $\mathbb{D}^{k, p}(\mathbb{R}, \gamma)$ the Sobolev space of functions which are $k$ times weakly differentiable, such that together with their derivatives up to order $k$, they have finite moments of order $p$ with respect to the measure $\gamma$. Also, we denote by $d_{\text {TV }}$ and $d_{\mathrm{W}}$ the total variation and Wasserstein distances, respectively. Along the paper, $Z$ will denote a $N(0,1)$ random variable. Our first result is the following.

Theorem 1.1. Assume that $g \in L^{2}(\mathbb{R}, \gamma)$ has Hermite rank $d \geq 2$ and satisfies $A(g) \in$ $\mathbb{D}^{1,4}(\mathbb{R}, \gamma)$. Suppose that (1.2) holds true and let $Y_{n}$ be the random variable defined in (1.5). Then we have the following estimates:

(i) If $d=2$, then

$$
d_{\mathrm{TV}}\left(Y_{n}, Z\right) \leq C n^{-\frac{1}{2}}\left(\sum_{|k| \leq n}|\rho(k)|\right)^{\frac{1}{2}}+C n^{-\frac{1}{2}}\left(\sum_{|k| \leq n}|\rho(k)|^{\frac{4}{3}}\right)^{\frac{3}{2}} .
$$

(ii) If $d \geq 3$, we have

$$
\begin{aligned}
d_{\mathrm{TV}}\left(Y_{n}, Z\right) \leq & C n^{-\frac{1}{2}} \sum_{|k| \leq n}|\rho(k)|^{d-1}\left(\sum_{|k| \leq n}|\rho(k)|^{2}\right)^{\frac{1}{2}} \\
& +C n^{-\frac{1}{2}}\left(\sum_{|k| \leq n}|\rho(k)|^{2}\right)^{\frac{1}{2}}\left(\sum_{|k| \leq n}|\rho(k)|\right)^{\frac{1}{2}} .
\end{aligned}
$$

The proof of these results is based on Proposition 2.1, that requires the estimation of $\operatorname{Var}\left(\left\langle D F_{n}, u_{n}\right\rangle_{\mathfrak{H}}\right)$, where $u_{n}$ is such that $F_{n}=\delta\left(u_{n}\right)$. Here $D$ and $\delta$ are the derivative and divergence operators associated with the Malliavin calculus for the Gaussian sequence $X$. Following the ideas developed in [8] and [17], we construct the sequence $u_{n}$ using the operator $T_{1}(g)$ that shifts in one unit the Hermite expansion of $g$. A basic ingredient of the proof is an explicit computation of the variance $\operatorname{Var}\left(\left\langle D F_{n}, u_{n}\right\rangle_{\mathfrak{H}}\right)$, using Wiener chaos expansions. For this we need a result on the convergence in $L^{2}$ of powers of truncated Wiener chaos expansions established in Proposition 3.1, which has its own interest. A sufficient condition for a function $g$ to satisfy $A(g) \in \mathbb{D}^{k, M}(\mathbb{R}, \gamma)$ for any integer $k \geq 0, M \geq 3$ is given in Lemma 3.3.

Let us compare Theorem 1.1 with the existing results in the literature. For $d=2$, the estimate (1.7) coincides with the estimate obtained in [17] (see Theorem 4.3 (iii)), assuming $g \in \mathbb{D}^{4,4}(\mathbb{R}, \gamma)$. This is the best estimate that one can obtain using Proposition 2.1 (it coincides with the bound for $g(x)=x^{2}-1$ ). In [17 this estimate is obtained applying Poincaré inequality to estimate the variance plus twice the integration-by-parts formula and 
for this reason one requires the function $g$ to be four times differentiable. Here, we only need one derivative, but for the function $A(g)$. In a recent note (see [12]), the authors have obtained the weaker bound

$$
d_{\mathrm{TV}}\left(Y_{n}, Z\right) \leq C n^{-\frac{1}{2}}\left(\sum_{|k| \leq n}|\rho(k)|\right)^{\frac{3}{2}}
$$

assuming only $g \in \mathbb{D}^{1,4}(\mathbb{R}, \gamma)$ and applying Gebelein's inequality, instead of Poincaré's inequality, to estimate the variance of $\left\langle D F_{n}, u_{n}\right\rangle_{\mathfrak{H}}$. Notice that the bound (1.9) holds, for example, for the function $g(x)=|x|-\mathbb{E}(|Z|)$, which belongs to $\mathbb{D}^{1,4}(\mathbb{R}, \gamma)$.

In the case $d \geq 3$, the estimate (1.8) coincides with the estimate obtained in 17, Theorem $4.5]$, assuming $g \in \mathbb{D}^{3 d-2,4}(\mathbb{R}, \gamma)$, and applying the integration-by-parts argument several times. Again our estimate requires only one derivative (for $A(g)$ ) instead of $3 d-2$ derivatives. Also, computing the third and fourth cumulants in the case $g=H_{d}$, leads to the optimal bound (see [2])

$$
d_{\mathrm{TV}}\left(Y_{n}, Z\right) \leq \frac{C}{n}\left(\sum_{|k| \leq n}|\rho(k)|^{d-1}\right)^{2} \sum_{|k| \leq n}|\rho(k)|^{2}+\frac{C}{\sqrt{n}}\left(\sum_{|k| \leq n}|\rho(k)|^{\frac{3 d}{4}}\right)^{2} \mathbf{1}_{\{d \text { even }\}} .
$$

The second part of the paper is devoted to showing two improvements of the above bound for $d=2$. First we establish the following upper bound for the Wasserstein distance, using a new estimate (see Proposition 2.3) and the representation of $F_{n}$ as an iterated divergence $F_{n}=\delta^{2}\left(v_{n}\right)$.

Theorem 1.2. Assume that $g \in L^{2}(\mathbb{R}, \gamma)$ has Hermite rank $d=2$ and satisfies $A(g) \in$ $\mathbb{D}^{2,6}(\mathbb{R}, \gamma)$. Suppose that (1.2) holds true and let $Y_{n}$ be the random variable defined in (1.5). Then we have the following estimate

$$
d_{\mathrm{W}}\left(Y_{n}, Z\right) \leq C n^{-\frac{1}{2}}\left(\sum_{|k| \leq n}|\rho(k)|\right)^{\frac{1}{2}}+C n^{-\frac{1}{2}}\left(\sum_{|k| \leq n}|\rho(k)|^{\frac{3}{2}}\right)^{2} .
$$

Going back to the total variation distance, we recall first that the optimal bound for $d=2$ is

$$
d_{\mathrm{TV}}\left(Y_{n}, Z\right) \leq C n^{-\frac{1}{2}}\left(\sum_{|k| \leq n}|\rho(k)|^{\frac{3}{2}}\right)^{2}
$$

This estimate was obtained for $g=H_{2}$ in [11, with a matching lower bound, and it was extended to $g \in \mathbb{D}^{6,8}(\mathbb{R}, \gamma)$ in [17]. This upper bound, however, cannot be obtained as a consequence of Proposition 2.1 and requires a more intensive application of Stein's method (see [11, 17]). Using Proposition 2.2, we have obtained the following result.

Theorem 1.3. Assume that $g \in L^{2}(\mathbb{R}, \gamma)$ has Hermite rank $d=2$ and satisfies $A(g) \in$ $\mathbb{D}^{3,8}(\mathbb{R}, \gamma)$. Suppose that (1.2) holds true and let $Y_{n}$ be the random variable defined in (1.5). Then the estimate (1.11) holds true.

Notice that the first term in (1.10) coincides with the first term in (1.7), while the second term is precisely the optimal rate for the total variation distance (1.11). 
The paper is organized as follows. Section 2 reviews some preliminaries on the Malliavin calculus for an isonormal Gaussian process and Stein's method. Section 3 presents a new result on the convergence in $L^{2}(\Omega)$ of powers of Wiener chaos expansions, which has its own interest. Finally, Sections 4, 5 and 6 contain the proofs of Theorems 1.1, 1.2 and 1.3, respectively.

Along the paper we will denote by $C$ a generic constant that may vary from line to line.

\section{Preliminaries}

In this section, we briefly recall some elements of the Malliavin calculus associated with a Gaussian family of random variables. We refer the reader to [10, 13, 14 for a detailed account on this topic. We will also recall two basic inequalities for the total variation distance proved using Stein's method and we present a new inequality for the Wasserstein distance.

2.1. Malliavin calculus. Let $\mathfrak{H}$ be a real separable Hilbert space. For any integer $m \geq 1$, we use $\mathfrak{H}^{\otimes m}$ and $\mathfrak{H}^{\odot m}$ to denote the $m$-th tensor product and the $m$-th symmetric tensor product of $\mathfrak{H}$, respectively. Let $W=\{W(\phi), \phi \in \mathfrak{H}\}$ denote an isonormal Gaussian process over the Hilbert space $\mathfrak{H}$. That means, $W$ is a centered Gaussian family of random variables, defined on some probability space $(\Omega, \mathcal{F}, P)$, with covariance

$$
\mathbb{E}(W(\phi) W(\psi))=\langle\phi, \psi\rangle_{\mathfrak{H}}, \quad \phi, \psi \in \mathfrak{H} .
$$

We assume that $\mathcal{F}$ is generated by $W$.

We denote by $\mathcal{H}_{m}$ the closed linear subspace of $L^{2}(\Omega)$ generated by the random variables $\left\{H_{m}(W(\varphi)): \varphi \in \mathfrak{H},\|\varphi\|_{\mathfrak{H}}=1\right\}$, where $H_{m}$ is the $m$-th Hermite polynomial defined by

$$
H_{m}(x)=(-1)^{m} e^{\frac{x^{2}}{2}} \frac{d^{m}}{d x^{m}} e^{-\frac{x^{2}}{2}}, \quad m \geq 1,
$$

and $H_{0}(x)=1$. The space $\mathcal{H}_{m}$ is called the Wiener chaos of order $m$. The $m$-th multiple integral of $\phi^{\otimes m} \in \mathfrak{H}^{\odot m}$ is defined by the identity $I_{m}\left(\phi^{\otimes m}\right)=H_{m}(W(\phi))$ for any $\phi \in \mathfrak{H}$ with $\|\phi\|_{\mathfrak{H}}=1$. The map $I_{m}$ provides a linear isometry between $\mathfrak{H}^{\odot m}$ (equipped with the norm $\sqrt{m !}\|\cdot\|_{\mathfrak{H}^{\otimes m}}$ ) and $\mathcal{H}_{m}$ (equipped with $L^{2}(\Omega)$ norm). By convention, $\mathcal{H}_{0}=\mathbb{R}$ and $I_{0}(x)=x$.

The space $L^{2}(\Omega)$ can be decomposed into the infinite orthogonal sum of the spaces $\mathcal{H}_{m}$. Namely, for any square integrable random variable $F \in L^{2}(\Omega)$, we have the following expansion,

$$
F=\sum_{m=0}^{\infty} I_{m}\left(f_{m}\right),
$$

where $f_{0}=\mathbb{E}(F)$, and $f_{m} \in \mathfrak{H}^{\odot m}$ are uniquely determined by $F$. This is known as the Wiener chaos expansion.

For a smooth and cylindrical random variable $F=f\left(W\left(\varphi_{1}\right), \ldots, W\left(\varphi_{n}\right)\right)$, with $\varphi_{i} \in \mathfrak{H}$ and $f \in C_{b}^{\infty}\left(\mathbb{R}^{n}\right)$ ( $f$ and its partial derivatives are bounded), we define its Malliavin derivative as the $\mathfrak{H}$-valued random variable given by

$$
D F=\sum_{i=1}^{n} \frac{\partial f}{\partial x_{i}}\left(W\left(\varphi_{1}\right), \ldots, W\left(\varphi_{n}\right)\right) \varphi_{i} .
$$

By iteration, we can also define the $k$-th derivative $D^{k} F$, which is an element in the space $L^{2}\left(\Omega ; \mathfrak{H}^{\otimes k}\right)$. For any real $p \geq 1$ and any integer $k \geq 1$, the Sobolev space $\mathbb{D}^{k, p}$ is defined as 
the closure of the space of smooth and cylindrical random variables with respect to the norm $\|\cdot\|_{k, p}$ defined by

$$
\|F\|_{k, p}^{p}=\mathbb{E}\left(|F|^{p}\right)+\sum_{i=1}^{k} \mathbb{E}\left(\left\|D^{i} F\right\|_{\mathfrak{H} \otimes i}^{p}\right) .
$$

We define the divergence operator $\delta$ as the adjoint of the derivative operator $D$. Namely, an element $u \in L^{2}(\Omega ; \mathfrak{H})$ belongs to the domain of $\delta$, denoted by Dom $\delta$, if there is a constant $c_{u}>0$ depending on $u$ and satisfying

$$
\left|\mathbb{E}\left(\langle D F, u\rangle_{\mathfrak{H}}\right)\right| \leq c_{u}\|F\|_{L^{2}(\Omega)}
$$

for any $F \in \mathbb{D}^{1,2}$. If $u \in \operatorname{Dom} \delta$, the random variable $\delta(u)$ is defined by the duality relationship

$$
\mathbb{E}(F \delta(u))=\mathbb{E}\left(\langle D F, u\rangle_{\mathfrak{H}}\right),
$$

which is valid for all $F \in \mathbb{D}^{1,2}$. In a similar way, for each integer $k \geq 2$, we define the iterated divergence operator $\delta^{k}$ through the duality relationship

$$
\mathbb{E}\left(F \delta^{k}(u)\right)=\mathbb{E}\left(\left\langle D^{k} F, u\right\rangle_{\mathfrak{H}^{\otimes k}}\right),
$$

valid for any $F \in \mathbb{D}^{k, 2}$, where $u \in \operatorname{Dom} \delta^{k} \subset L^{2}\left(\Omega ; \mathfrak{H}^{\otimes k}\right)$.

Let $\gamma$ be the standard Gaussian measure on $\mathbb{R}$. The Hermite polynomials $\left\{H_{m}(x), m \geq 0\right\}$ form a complete orthonormal system in $L^{2}(\mathbb{R}, \gamma)$ and any function $g \in L^{2}(\mathbb{R}, \gamma)$ admits an orthogonal expansion of the form (1.1). If $g$ has Hermite rank $d$, for any integer $1 \leq k \leq d$, we define the operator $T_{k}$ by

$$
T_{k}(g)(x)=\sum_{m=d}^{\infty} c_{m} H_{m-k}(x) .
$$

To simplify the notation we will write $T_{k}(g)=g_{k}$.

Suppose that $F$ is a random variable in the first Wiener chaos of $W$ of the form $F=I_{1}(\varphi)$, where $\varphi \in \mathfrak{H}$ has norm one. Then $g_{k}(F)$ has the representation

$$
g(F)=\delta^{k}\left(g_{k}(F) \varphi^{\otimes k}\right) .
$$

Moreover, if $g(F) \in \mathbb{D}^{j, p}$ for some $j \geq 0$ and $p>1$, then $g_{k}(F) \in \mathbb{D}^{j+k, p}$. We refer to [17] for the proof of these results.

Consider $\mathfrak{H}=\mathbb{R}$, the probability space $(\Omega, \mathcal{F}, P)=(\mathbb{R}, \mathcal{B}(\mathbb{R}), \gamma)$ and the isonormal Gaussian process $W(h)=h$. For any $k \geq 0$ and $p \geq 1$, denote by $\mathbb{D}^{k, p}(\mathbb{R}, \gamma)$ the corresponding Sobolev spaces of functions. Notice that if $F=I_{1}(\varphi)$ is an element in the first Wiener chaos with $\|\varphi\|_{\mathfrak{H}}=1$, then $g \in \mathbb{D}^{k, p}(\mathbb{R}, \gamma)$ if and only if $g(F) \in \mathbb{D}^{k, p}$.

2.2. Stein's method. We refer to [6] for a complete presentation of this topic. Let $h: \mathbb{R} \rightarrow \mathbb{R}$ be a Borel function such that $h \in L^{1}(\mathbb{R}, \gamma)$. The ordinary differential equation

$$
f^{\prime}(x)-x f(x)=h(x)-\mathbb{E}(h(Z))
$$

is called the Stein's equation associated with $h$. The function

$$
f_{h}(x):=e^{x^{2} / 2} \int_{-\infty}^{x}(h(y)-\mathbb{E}(h(Z))) e^{-y^{2} / 2} d y
$$

is the unique solution to the Stein's equation satisfying $\lim _{|x| \rightarrow \infty} e^{-x^{2} / 2} f_{h}(x)=0$. Moreover, if $h$ is bounded by $1, f_{h}$ satisfies $\left\|f_{h}\right\|_{\infty} \leq \sqrt{\pi / 2}$ and $\left\|f_{h}^{\prime}\right\|_{\infty} \leq 2$. On the other hand, if $h \in \operatorname{Lip}(1)$ ( $h$ is Lipschitz with a Lipschitz constant bounded by 1 ), then $f_{h}$ is continuously 
differentiable, $\left\|f_{h}^{\prime}\right\|_{\infty} \leq \sqrt{2 / \pi}$ and (see [19, Lemma 3]) $\left\|f_{h}^{\prime \prime}\right\|_{\infty} \leq 2$. We refer to [10] and the references therein for a complete proof of these results.

We recall that the total variation distance between the laws of two random variables $F, G$ is defined by

$$
d_{\mathrm{TV}}(F, G)=\sup _{B \in \mathcal{B}(\mathbb{R})}|P(F \in B)-P(G \in B)|,
$$

where the supremum runs over all Borel sets $B \subset \mathbb{R}$. Substituting $x$ by $F$ in Stein's equation (2.6) and using the estimate for $\left\|f_{h}^{\prime}\right\|_{\infty}$ lead to the fundamental estimate

$$
d_{\mathrm{TV}}(F, Z) \leq \sup _{f \in \mathcal{C}^{1}(\mathbb{R}),\left\|f^{\prime}\right\|_{\infty} \leq 2}\left|\mathbb{E}\left(f^{\prime}(F)-F f(F)\right)\right| .
$$

Furthermore, the Wasserstein distance between the laws of two random variables $F, G$ is defined by

$$
d_{\mathrm{W}}(F, G)=\sup _{f \in \operatorname{Lip}(1)} \mid \mathbb{E}(f(F))-\mathbb{E}(f((G)) \mid
$$

and using Stein's equation leads to

$$
d_{\mathrm{W}}(F, G) \leq \sup _{f \in \mathcal{F}_{W}}\left|\mathbb{E}\left(f^{\prime}(F)-F f(F)\right)\right|,
$$

where $\mathcal{F}_{W}$ is the set of functions $f \in \mathcal{C}^{2}(\mathbb{R})$ such that $\left\|f_{h}^{\prime}\right\|_{\infty} \leq \sqrt{2 / \pi}$ and $\left\|f_{h}^{\prime \prime}\right\|_{\infty} \leq 2$.

In the framework of an isonormal Gaussian process $W$, we can use Stein's equation to estimate the total variation distance between a random variable $F=\delta(u)$ and $Z$. A basic result is given in the next proposition (see [15, 10]), which is an easy consequence of (2.7) and the duality relationship (2.2).

Proposition 2.1. Assume that $u \in \operatorname{Dom} \delta, F=\delta(u) \in \mathbb{D}^{1,2}$ and $\mathbb{E}\left(F^{2}\right)=1$. Then,

$$
d_{\mathrm{TV}}(F, Z) \leq 2 \sqrt{\operatorname{Var}\left(\langle D F, u\rangle_{\mathfrak{H}}\right)} .
$$

An iterative application of the Stein-Malliavin approach leads to the following result, which requires the random variable $F$ to be three times differentiable (see [17, Proposition 3.2.]).

Proposition 2.2. Assume that $u \in \operatorname{Dom} \delta, F=\delta(u) \in \mathbb{D}^{3,2}$ and $\mathbb{E}\left(F^{2}\right)=1$. Then,

$$
d_{T V}(F, Z) \leq(8+\sqrt{32 \pi}) \operatorname{Var}\left(\langle D F, u\rangle_{\mathfrak{H}}\right)+\sqrt{2 \pi}\left|\mathbb{E}\left(F^{3}\right)\right|+\sqrt{32 \pi} \mathbb{E}\left(\left|D_{u} F\right|^{2}\right)+4 \pi \mathbb{E}\left(\left|D_{u}^{3} F\right|\right),
$$

where we have used the notation $D_{u} F=\langle u, D F\rangle_{\mathfrak{H}}$ and $D_{u}^{i+1} F=\left\langle u, D\left(D_{u}^{i} F\right)\right\rangle_{\mathfrak{H}}$ for $i \geq 1$.

In the next proposition we present a new estimate for the Wasserstein's distance between a random variable $F=\delta^{2}(v)$ and a $N(0,1)$ random variable obtained using Stein's method and Malliavin calculus.

Proposition 2.3. Assume that $v \in \operatorname{Dom} \delta^{2}, F=\delta^{2}(v) \in \mathbb{D}^{2,2}$ and $\mathbb{E}\left(F^{2}\right)=1$. Then,

$$
d_{W}(F, Z) \leq \sqrt{2 / \pi} \sqrt{\operatorname{Var}\left(\left\langle D^{2} F, v\right\rangle_{\mathfrak{H}^{\otimes 2}}\right)}+2 \mathbb{E}\left(\left|\langle D F \otimes D F, v\rangle_{\mathfrak{H}^{\otimes 2}}\right|\right)
$$


Proof. By the duality relation (2.3), $\mathbb{E}\left(F \delta^{2}(v)\right)=\mathbb{E}\left(\left\langle D^{2} F, v\right\rangle_{\mathfrak{H}^{\otimes 2}}\right)$. As a consequence, using (2.8) we can write

$$
\begin{aligned}
d_{W}(F, Z) & \leq \sup _{f \in \mathcal{F}_{W}}\left|\mathbb{E}\left(f^{\prime}(F)\right)-\mathbb{E}(F f(F))\right|=\sup _{f \in \mathcal{F}_{W}}\left|\mathbb{E}\left(f^{\prime}(F)\right)-\mathbb{E}\left(\delta^{2}(v) f(F)\right)\right| \\
& =\sup _{f \in \mathcal{F}_{W}}\left|\mathbb{E}\left(f^{\prime}(F)\right)-\mathbb{E}\left(\left\langle D^{2}(f(F)), v\right\rangle_{\mathfrak{H}^{\otimes 2}}\right)\right| \\
& =\sup _{f \in \mathcal{F}_{W}}\left|\mathbb{E}\left(f^{\prime}(F)\right)-\mathbb{E}\left(f^{\prime}(F)\left\langle D^{2} F, v\right\rangle_{\mathfrak{H}^{\otimes 2}}\right)-\mathbb{E}\left(f^{\prime \prime}(F)\langle D F \otimes D F, v\rangle_{\mathfrak{H}^{\otimes 2}}\right)\right| \\
& \leq \sqrt{2 / \pi} \mathbb{E}\left(\left|1-\left\langle D^{2} F, v\right\rangle_{\mathfrak{H}^{\otimes 2}}\right|\right)+2 \mathbb{E}\left(\left|\langle D F \otimes D F, v\rangle_{\mathfrak{H}^{\otimes 2}}\right|\right) .
\end{aligned}
$$

Now, since $1=\mathbb{E}\left(F^{2}\right)=\mathbb{E}\left(F \delta^{2}(v)\right)=\mathbb{E}\left(\left\langle D^{2} F, v\right\rangle_{\mathfrak{H}^{\otimes 2}}\right)$, using Cauchy-Schwarz inequality, we get

$$
\mathbb{E}\left(\left|1-\left\langle D^{2} F, v\right\rangle_{\mathfrak{H}^{\otimes 2}}\right|\right) \leq \sqrt{\mathbb{E}\left(\left|\mathbb{E}\left(\left\langle D^{2} F, v\right\rangle_{\mathfrak{H}^{\otimes 2}}\right)-\left\langle D^{2} F, v\right\rangle_{\mathfrak{H}^{\otimes 2}}\right|^{2}\right)}=\sqrt{\operatorname{Var}\left(\left\langle D^{2} F, v\right\rangle_{\mathfrak{H}^{\otimes 2}}\right)},
$$

which concludes our proof.

2.3. Some basic inequalities. In this subsection we recall several inequalities proved in [17] (see Lemmas 6.6, 6.7 and 6.8), which can be deduced from the Brascamp-Lieb inequality (see [3]) or just using Hölder's and Young's convolution inequalities.

Lemma 2.1. Fix an integer $M \geq 2$. Let $f$ be a non-negative function on the integers and set $\mathbf{k}=\left(k_{1}, \ldots, k_{M}\right)$. Then, we have:

(i) For any vector $\mathbf{v} \in \mathbb{R}^{M}$ whose components are 1 or -1

$$
\sum_{\mathbf{k} \in \mathbb{Z}^{M}} f(\mathbf{k} \cdot \mathbf{v}) \prod_{j=1}^{M} f\left(k_{j}\right) \leq C\left(\sum_{k \in \mathbb{Z}} f(k)^{1+\frac{1}{M}}\right)^{M} .
$$

(ii) For any vector $\mathbf{v} \in \mathbb{R}^{M}$ whose components are 0,1 or -1 , assuming $\sum_{k \in \mathbb{Z}} f(k)^{2}<\infty$,

$$
\sum_{\mathbf{k} \in \mathbb{Z}^{M}} f(\mathbf{k} \cdot \mathbf{v}) \prod_{j=1}^{M} f\left(k_{j}\right) \leq C\left(\sum_{k \in \mathbb{Z}} f(k)\right)^{M-1} .
$$

(iii) Suppose $M \geq 3$. Let $\mathbf{v}, \mathbf{w} \in \mathbb{R}^{M}$ be linearly independent vectors, whose components are 0,1 or -1 . Suppose $\sum_{k \in \mathbb{Z}} f(k)^{2}<\infty$. Then,

$$
\sum_{\mathbf{k} \in \mathbb{Z}^{M}} f(\mathbf{k} \cdot \mathbf{v}) f(\mathbf{k} \cdot \mathbf{w}) \prod_{j=1}^{M} f\left(k_{j}\right) \leq C\left(\sum_{k \in \mathbb{Z}} f(k)\right)^{M-2} .
$$

\section{Some Remarks on Wiener chaOs expansions}

In this section we present some useful results on Wiener chaos expansions. We first recall a formula for the expectation of the product of multiple stochastic integrals.

Lemma 3.1. Let $q_{i} \geq 1$ be integers, and consider functions $f_{i} \in \mathfrak{H}^{\odot q_{i}}, i=1, \ldots, M$. Then,

$$
\mathbb{E}\left(\prod_{i=1}^{M} I_{q_{i}}\left(f_{i}\right)\right)=\sum_{\beta \in \mathcal{D}_{q}} C_{q, \beta}\left(\otimes_{i=1}^{M} f_{i}\right)_{\beta},
$$


where

$$
C_{q, \beta}=\frac{\prod_{i=1}^{M} q_{i} !}{\prod_{1 \leq j<k \leq M} \beta_{j k} !},
$$

$\mathcal{D}_{q}$ is the set of nonnegative integers $\beta_{j k}, 1 \leq j<k \leq M$ satisfying

$$
q_{i}=\sum_{j \text { or } k=i} \beta_{j k}, \quad i=1 \ldots, M
$$

and $\left(\otimes_{i=1}^{M} f_{i}\right)_{\beta}$ denotes the contraction of $\beta_{j k}$ indexes between $f_{j}$ and $f_{k}$, for all $1 \leq j<k \leq$ $M$.

Proof. The product formula for multiple stochastic integrals (see, for instance, [18, Theorem 6.1.1], or formula (2.1) in [1] for $M=2$ ) says that

$$
\prod_{i=1}^{M} I_{q_{i}}\left(f_{i}\right)=\sum_{\mathcal{P}, \psi} I_{\gamma_{1}+\cdots+\gamma_{M}}\left(\left(\otimes_{i=1}^{M} f_{i}\right)_{\mathcal{P}, \psi}\right),
$$

where $\mathcal{P}$ denotes the set of all partitions $\left\{1, \ldots, q_{i}\right\}=J_{i} \cup\left(\cup_{k=1, \ldots, M, k \neq i} I_{i k}\right)$, where for any $i, k=1, \ldots, M, I_{i k}$ and $I_{k i}$ have the same cardinality, $\psi_{i k}$ is a bijection between $I_{i k}$ and $I_{k i}$ and $\gamma_{i}=\left|J_{i}\right|$. Moreover, $\left(\otimes_{i=1}^{M} f_{i}\right)_{\mathcal{P}, \psi}$ denotes the contraction of the indexes $\ell$ and $\psi_{i k}(\ell)$ for any $\ell \in I_{i k}$ and any $i, k=1 \ldots, M$. Then, the expectation $\mathbb{E}\left(\prod_{i=1}^{M} I_{q_{i}}\left(f_{i}\right)\right)$ corresponds to the case $\gamma_{1}=\cdots=\gamma_{M}=0$, and, if we specify the number of partitions for fixed cardinalities $\beta_{j k}$, we obtain the desired formula.

3.1. Convergence of truncated expansions. In general, given a random variable $F \in$ $L^{2}(\Omega)$ with chaos expansion (2.1), the fact that $\mathbb{E}\left(|F|^{p}\right)<\infty$ for some $p>2$ does not imply that the chaos expansion converges in $L^{p}(\Omega)$. The next proposition provides a partial result in this direction for $p=2 M$ and in the one-dimensional case, assuming that all the coefficients are nonnegative.

Proposition 3.1. Consider a function $g \in L^{2}(\mathbb{R}, \gamma)$, with an expansion of the form $g(x)=$ $\sum_{q=0}^{\infty} c_{q} H_{q}(x)$. Suppose that $c_{q} \geq 0$ for each $q \geq 0$ and $g \in L^{2 M}(\mathbb{R}, \gamma)$ for some $M \geq 1$. Consider the truncated sequence

$$
g^{(N)}:=\sum_{q=0}^{N} c_{q} H_{q} .
$$

Then $\left(g^{(N)}\right)^{M}$ converges in $L^{2}(\mathbb{R}, \gamma)$ to $g^{M}$.

Proof. The proof will be done by induction on $M$. The result is clearly true for $M=1$. Suppose that $M \geq 2$ and the result holds for $M-1$. Using the product formula for Hermite polynomials, which is a particular case of (3.1), we can write

$$
\begin{aligned}
\left(g^{(N)}\right)^{M} & =\sum_{q_{1}, \ldots, q_{M}=0}^{N} \prod_{i=1}^{M} c_{q_{i}} H_{q_{i}} \\
& =\sum_{q_{1}, \ldots, q_{M}=0}^{N}\left(\prod_{i=1}^{M} c_{q_{i}}\right) \sum_{(\beta, \gamma) \in \widehat{\mathcal{D}}_{q}} C_{q, \beta, \gamma} H_{\gamma_{1}+\cdots+\gamma_{M}},
\end{aligned}
$$


where

$$
C_{q, \beta, \gamma}=\frac{\prod_{i=1}^{M} q_{i} !}{\prod_{i=1}^{M} \gamma_{i} ! \prod_{1 \leq j<k \leq M} \beta_{j k} !},
$$

and $\widehat{\mathcal{D}}_{q}$ is the set of nonnegative integers $\beta_{j k}, 1 \leq j<k \leq M$ and $\gamma_{i}, 1 \leq i \leq M$, satisfying

$$
q_{i}=\gamma_{i}+\sum_{j \text { or } k=i} \beta_{j k}, \quad i=1, \ldots, M .
$$

As a consequence, we obtain

where

$$
\left(g^{(N)}\right)^{M}=\sum_{m=0}^{\infty} d_{m, N} H_{m}
$$

$$
d_{m, N}=\sum_{q_{1}, \ldots, q_{M}=0}^{N}\left(\prod_{i=1}^{M} c_{q_{i}}\right) \sum_{(\beta, \gamma) \in \widehat{\mathcal{D}}_{q}, \gamma_{1}+\cdots+\gamma_{M}=m} C_{q, \beta, \gamma} .
$$

The function $g^{M}$ belongs to $L^{2}(\mathbb{R}, \gamma)$. Therefore, it will have an expansion of the form

$$
g^{M}=\sum_{m=0}^{\infty} d_{m} H_{m}
$$

In order to compute the coefficients $d_{m}$, taking into account that $g H_{m} \in L^{2}(\mathbb{R}, \gamma)$ and, by the induction hypothesis, $\left(g^{(N)}\right)^{M-1}$ converges to $g^{M-1}$ in $L^{2}(\mathbb{R}, \gamma)$ as $N \rightarrow \infty$, we can write

$$
d_{m}=\frac{1}{m !} \mathbb{E}\left(g^{M} H_{m}\right)=\lim _{N \rightarrow \infty} \frac{1}{m !} \mathbb{E}\left(g\left(g^{(N)}\right)^{M-1} H_{m}\right) .
$$

To compute the expectation $\mathbb{E}\left(g\left(g^{(N)}\right)^{M-1} H_{m}\right)$ we need the chaos expansion of $\left(g^{(N)}\right)^{M-1} H_{m}$ :

$$
\left(g^{(N)}\right)^{M-1} H_{m}=\sum_{q_{1}, \ldots, q_{M-1}=0}^{N} \prod_{i=1}^{M-1} c_{q_{i}} \sum_{\left(\beta^{\prime}, \gamma^{\prime}\right) \in \widehat{\mathcal{D}}_{q}^{\prime}} C_{q, \beta^{\prime}, \gamma^{\prime}} H_{\gamma_{1}^{\prime}+\cdots+\gamma_{M}^{\prime}},
$$

where

$$
C_{q, \beta^{\prime}, \gamma^{\prime}}=\frac{m ! \prod_{i=1}^{M-1} q_{i} !}{\prod_{i=1}^{M} \gamma_{i}^{\prime} ! \prod_{1 \leq j<k \leq M} \beta_{j k}^{\prime} !},
$$

and $\widehat{\mathcal{D}}_{q}^{\prime}$ is the set of $\beta$ 's and $\gamma$ 's such that (3.3) holds for $i=1, \ldots, M-1$ and

$$
m=\gamma_{M}+\sum_{j \text { or } k=M} \beta_{j k}^{\prime} \text {. }
$$

As a consequence,

$$
\mathbb{E}\left(g\left(g^{(N)}\right)^{M-1} H_{m}\right)=\sum_{q=0}^{\infty} q ! c_{q} \sum_{q_{1}, \ldots, q_{M-1}=0}^{N} \prod_{i=1}^{M-1} c_{q_{i}} \sum_{\left(\beta^{\prime} \gamma^{\prime}\right) \in \widehat{\mathcal{D}}_{q}^{\prime}, \gamma_{1}^{\prime}+\cdots+\gamma_{M}^{\prime}=q} C_{q, \beta^{\prime}, \gamma^{\prime}}
$$

and, taking into account that the coefficients $c_{q}$ are nonnegative and putting $q=q_{M}$,

$$
d_{m}=\sum_{q_{1}, \ldots, q_{M}=0}^{\infty} \prod_{i=1}^{M} c_{q_{i}} \sum_{\left(\beta^{\prime}, \gamma^{\prime}\right) \in \widehat{\mathcal{D}}_{q}^{\prime}, \gamma_{1}^{\prime}+\cdots+\gamma_{M}^{\prime}=q_{M}} \frac{\prod_{i=1}^{M} q_{i} !}{\prod_{i=1}^{M} \gamma_{i}^{\prime} ! \prod_{1 \leq j<k \leq M} \beta_{j k}^{\prime} !} .
$$


We claim that for any $\left(\beta^{\prime}, \gamma^{\prime}\right) \in \widehat{\mathcal{D}}_{q}^{\prime}$ there exist a unique element $(\beta, \gamma) \in \widehat{\mathcal{D}}_{q}$ such that

$$
\prod_{i=1}^{M} \gamma_{i} ! \prod_{1 \leq j<k \leq M} \beta_{j k} !=\prod_{i=1}^{M} \gamma_{i}^{\prime} ! \prod_{1 \leq j<k \leq M} \beta_{j k}^{\prime} ! .
$$

Indeed, it suffices to take $\beta_{j k}=\beta_{j k}^{\prime}$ if $1 \leq j<k \leq M-1, \gamma_{i}=\beta_{i M}^{\prime}$ for $i=1, \ldots, M-1$, $\gamma_{M}=\gamma_{M}^{\prime}$, and $\beta_{j M}=\gamma_{j}^{\prime}$ for $1 \leq j \leq M-1$. It follows that $\lim _{N \rightarrow \infty} d_{m, N}=d_{m}$. This implies that $\left(g^{(N)}\right)^{M}$ converges in $L^{2}(\mathbb{R}, \gamma)$ to $g^{M}$ and allows us to complete the proof.

3.2. The absolute value operator. Recall that $A$, defined in (1.6) is the operator acting on $L^{2}(\mathbb{R}, \gamma)$ which replace the Hermite coefficients by its absolute values. Clearly, for any integer $k \geq 0$, and for any $g \in \mathbb{D}^{k, 2}(\mathbb{R}, \gamma)$, we have

$$
\|A(g)\|_{k, 2}=\|g\|_{k, 2} \text {. }
$$

Therefore, $g$ belongs to $\mathbb{D}^{k, 2}(\mathbb{R}, \gamma)$ if and only if $A(g) \in \mathbb{D}^{k, 2}(\mathbb{R}, \gamma)$. If we consider functions in $L^{p}(\mathbb{R}, \gamma)$ for some real number $p>2$, we do not know whether $g \in L^{p}(\mathbb{R}, \gamma)$ implies $A(g) \in L^{p}(\mathbb{R}, \gamma)$. However, the following result holds.

Lemma 3.2. Suppose that $A(g) \in \mathbb{D}^{k, 2 M}(\mathbb{R}, \gamma)$ for some integers $M \geq 2$ and $k \geq 0$. Then $g \in \mathbb{D}^{k, 2 M}(\mathbb{R}, \gamma)$.

Proof. We will show the result only for $k=0$, the case $k \geq 1$ being similar. Let $g=\sum_{q=d}^{\infty} c_{q} H_{q}$ and define $g_{+}=\sum_{q=d}^{\infty} c_{q} \mathbf{1}_{\left\{q: c_{q}>0\right\}} H_{q}$ and $g_{-}=\sum_{q=d}^{\infty} c_{q} \mathbf{1}_{\left\{q: c_{q}<0\right\}} H_{q}$. Then $g=g_{+}+g_{-}$. We will show that $g_{+} \in L^{2 M}(\mathbb{R}, \gamma)$, and in the same way one can prove that $g_{-} \in L^{2 M}(\mathbb{R}, \gamma)$. Using Proposition 3.1, we can write

$$
\begin{aligned}
\mathbb{E}\left(g_{+}^{2 M}\right) & =\lim _{N \rightarrow \infty} \mathbb{E}\left(\left(g_{+}^{(N)}\right)^{2 M}\right) \\
& =\sum_{q_{1}, \ldots, q_{2 M}=0}^{\infty}\left(\prod_{i=1}^{2 M} c_{q_{i}} \mathbf{1}_{\left\{q: c_{q}>0\right\}}\right) \sum_{\beta \in \mathcal{D}_{q}} \frac{\prod_{i=1}^{2 M} q_{i} !}{\prod_{1 \leq j<k \leq 2 M} \beta_{j k} !},
\end{aligned}
$$

where $\mathcal{D}_{q}$ is the set of nonnegative integers $\beta_{j k}, 1 \leq j<k \leq 2 M$, satisfying $q_{i}=\sum_{j \text { or } k=i} \beta_{j k}$, $i=1, \ldots, 2 M$. Clearly, this implies that $\mathbb{E}\left(g_{+}^{2 M}\right) \leq \mathbb{E}\left(A(g)^{2 M}\right)<\infty$.

The next lemma provides a criterion for a function $g$ to satisfy $A(g) \in \mathbb{D}^{\ell, M}(\gamma)$ for integers $\ell \geq 0, M \geq 3$.

Lemma 3.3. Fix integers $\ell \geq 0$ and $M \geq 3$. Let $g$ be a function in $g \in \mathbb{D}^{\ell, 2}(\mathbb{R}, \gamma)$, with Hermite expansion $g=\sum_{k=0}^{\infty} c_{q} H_{q}$. Then, $A(g) \in \mathbb{D}^{\ell, M}(\mathbb{R}, \gamma)$ if

$$
\sum_{q=0}^{\infty}\left|c_{q}\right| q^{\frac{\ell}{2}-\frac{1}{4}} \sqrt{q !}(M-1)^{\frac{q}{2}}<\infty
$$

Proof. We have

$$
D^{\ell} A^{(N)}(g)=\sum_{q=\ell}^{N}\left|c_{q}\right| q(q-1) \cdots(q-\ell+1) H_{q-\ell} .
$$

Applying the estimate (see, for instance, [7])

$$
\left\|H_{q}\right\|_{L^{M}(\mathbb{R}, \gamma)}=c(M) q^{-\frac{1}{4}} \sqrt{q !}(M-1)^{\frac{q}{2}}\left(1+O\left(q^{-1}\right)\right)
$$


we obtain

$$
\begin{aligned}
\left\|D^{\ell} A^{(N)}(g)\right\|_{L^{M}(\mathbb{R}, \gamma) \leq} & c(M)\left(\left|c_{\ell}\right| \sum_{q=\ell}^{N}\left|c_{q}\right| q(q-1) \cdots(q-\ell+1)(q-\ell)^{-\frac{1}{4}}\right. \\
& \left.\times \sqrt{(q-\ell) !}(M-1)^{\frac{q-\ell}{2}}\left(1+O\left(q^{-1}\right)\right)\right) \\
\leq & c(M, \ell)\left(\left|c_{\ell}\right|+\sum_{q=\ell}^{N}\left|c_{q}\right| q^{\frac{\ell}{2}-\frac{1}{4}} \sqrt{q !}(M-1)^{\frac{q-\ell}{2}}\left(1+O\left(q^{-1}\right)\right)\right) .
\end{aligned}
$$

Therefore, taking into account that $A^{(N)}(g)$ converges in $L^{2}(\Omega)$ to $A(g)$ as $N$ tends to infinity, we conclude that $\mathbb{E}\left(\left|D^{\ell} A(g)\right|^{M}\right)<\infty$ if (3.4) holds.

\section{Proof of Theorem 1.1}

Proof. Consider a centered stationary Gaussian family of random variables $X=\left\{X_{n}, n \geq 0\right\}$ with unit variance and covariance $\rho(k)=\mathbb{E}\left(X_{0} X_{k}\right)$ for $k \geq 0$. We put $\rho(-k)=\rho(k)$ for $k<0$. Suppose that $\mathfrak{H}$ is a Hilbert space and $e_{i} \in \mathfrak{H}, i \geq 0$, are elements such that, for each $i, j \geq 0$, we have $\left\langle e_{i}, e_{j}\right\rangle_{\mathfrak{H}}=\rho(i-j)$. In this situation, if $\{W(\phi): \phi \in \mathfrak{H}\}$ is an isonormal Gaussian process, then the sequence $X=\left\{X_{n}, n \geq 0\right\}$ has the same law as $\left\{W\left(e_{n}\right), n \geq 0\right\}$ and we can assume, without any loss of generality, that $X_{n}=W\left(e_{n}\right)$.

Consider the sequence $F_{n}:=\frac{1}{\sqrt{n}} \sum_{j=1}^{n} g\left(X_{j}\right)$ introduced in (1.5), where $g \in L^{2}(\mathbb{R}, \gamma)$ has Hermite rank $d \geq 2$ and let $\sigma_{n}^{2}=\mathbb{E}\left(F_{n}^{2}\right)$. Under condition (1.2), it is well known that as $n \rightarrow \infty, \sigma_{n}^{2} \rightarrow \sigma^{2}$, where $\sigma^{2}$ has been defined in (1.4). Set $Y_{n}=\frac{F_{n}}{\sigma_{n}}$. Notice that $\sigma>0$ implies that $\sigma_{n}$ is bounded below for $n$ large enough. Taking into account (2.5), we have the representation $Y_{n}=\delta\left(\frac{1}{\sigma_{n}} u_{n}\right)$, where

$$
u_{n}=\frac{1}{\sqrt{n}} \sum_{j=1}^{n} g_{1}\left(X_{j}\right) e_{j},
$$

and $g_{1}$ is the shifted function introduced in (2.4).

As a consequence of Proposition 2.1, we have the estimate

$$
\begin{aligned}
d_{T V}\left(Y_{n}, Z\right) & \leq 2 \sqrt{\operatorname{Var}\left(\left\langle D Y_{n}, \frac{1}{\sigma_{n}} u_{n}\right\rangle_{\mathfrak{H}}\right)} \\
& \leq C \sqrt{\operatorname{Var}\left(\left\langle D F_{n}, u_{n}\right\rangle_{\mathfrak{H}}\right)} .
\end{aligned}
$$

Then, we can write

$$
\left\langle D F_{n}, u_{n}\right\rangle_{\mathfrak{H}}=\frac{1}{n} \sum_{i, j=1}^{n} g^{\prime}\left(X_{i}\right) g_{1}\left(X_{j}\right) \rho(i-j) .
$$

The random variable $g^{\prime}\left(X_{i}\right) g_{1}\left(X_{j}\right)$ belongs to $L^{2}(\Omega)$, but we do not know its chaos expansion. For this reason, we need to use a limit argument. We have

$$
\left\langle D F_{n}, u_{n}\right\rangle_{\mathfrak{H}}=\lim _{N \rightarrow \infty} \Phi_{n, N}
$$


where the convergence holds in $L^{1}(\Omega)$ and

$$
\Phi_{n, N}=\frac{1}{n} \sum_{i, j=1}^{n} \sum_{q_{1}, q_{2}=d}^{N} c_{q_{1}} c_{q_{2}} q_{1} H_{q_{1}-1}\left(X_{i}\right) H_{q_{2}-1}\left(X_{j}\right) \rho(i-j) .
$$

Therefore, by Fatou's lemma

$$
\begin{aligned}
\operatorname{Var}\left(\left\langle D F_{n}, u_{n}\right\rangle_{\mathfrak{H}}\right) & =\mathbb{E}\left(\left\langle D F_{n}, u_{n}\right\rangle_{\mathfrak{H}}^{2}\right)-\left(\mathbb{E}\left(\left\langle D F_{n}, u_{n}\right\rangle_{\mathfrak{H}}\right)\right)^{2} \\
& \leq \liminf _{N \rightarrow \infty}\left(\mathbb{E}\left(\Phi_{n, N}^{2}\right)-\left(\mathbb{E}\left(\Phi_{n, N}\right)\right)^{2}\right) \\
& =\liminf _{N \rightarrow \infty} \operatorname{Var}\left(\Phi_{n, N}\right) .
\end{aligned}
$$

We can write

$$
\begin{aligned}
\operatorname{Var}\left(\Phi_{n, N}\right)= & \frac{1}{n^{2}} \sum_{i_{1}, i_{2}, i_{3}, i_{4}=1}^{n} \sum_{q_{1}, q_{2}, q_{3}, q_{4}=d}^{N} q_{1} q_{3} c_{q_{1}} c_{q_{2}} c_{q_{3}} c_{q_{4}} \rho\left(i_{1}-i_{2}\right) \rho\left(i_{3}-i_{4}\right) \\
& \times \operatorname{Cov}\left(H_{q_{1}-1}\left(X_{i_{1}}\right) H_{q_{2}-1}\left(X_{i_{2}}\right), H_{q_{3}-1}\left(X_{i_{3}}\right) H_{q_{4}-1}\left(X_{i_{4}}\right)\right) .
\end{aligned}
$$

The next step is to compute the covariance appearing in the previous formula. To do this we will write the Hermite polynomials in terms of stochastic integrals and apply Lemma 3.1. That is,

$$
\begin{aligned}
& \operatorname{Cov}\left(H_{q_{1}-1}\left(X_{i_{1}}\right) H_{q_{2}-1}\left(X_{i_{2}}\right), H_{q_{3}-1}\left(X_{i_{3}}\right) H_{q_{4}-1}\left(X_{i_{4}}\right)\right) \\
& =\operatorname{Cov}\left(I_{q_{1}-1}\left(e_{i_{1}}^{\otimes\left(q_{1}-1\right)}\right) I_{q_{2}-1}\left(e_{i_{2}}^{\otimes\left(q_{2}-1\right)}\right), I_{q_{3}-1}\left(e_{i_{3}}^{\otimes\left(q_{3}-1\right)}\right) I_{q_{4}-1}\left(e_{i_{4}}^{\otimes\left(q_{4}-1\right)}\right)\right) \\
& =\mathbb{E}\left(I_{q_{1}-1}\left(e_{i_{1}}^{\otimes\left(q_{1}-1\right)}\right) I_{q_{2}-1}\left(e_{i_{2}}^{\otimes\left(q_{2}-1\right)}\right) I_{q_{3}-1}\left(e_{i_{3}}^{\otimes\left(q_{3}-1\right)}\right) I_{q_{4}-1}\left(e_{i_{4}}^{\otimes\left(q_{4}-1\right)}\right)\right) \\
& \quad-\mathbb{E}\left(I_{q_{1}-1}\left(e_{i_{1}}^{\otimes\left(q_{1}-1\right)}\right) I_{q_{2}-1}\left(e_{i_{2}}^{\otimes\left(q_{2}-1\right)}\right)\right) \mathbb{E}\left(I_{q_{3}-1}\left(e_{i_{3}}^{\otimes\left(q_{3}-1\right)}\right) I_{q_{4}-1}\left(e_{i_{4}}^{\otimes\left(q_{4}-1\right)}\right)\right)
\end{aligned}
$$

and using Lemma 3.1 .

$$
\begin{aligned}
& \mathbb{E}\left(I_{q_{1}-1}\left(e_{i_{1}}^{\otimes\left(q_{1}-1\right)}\right) I_{q_{2}-1}\left(e_{i_{2}}^{\otimes\left(q_{2}-1\right)}\right) I_{q_{3}-1}\left(e_{i_{3}}^{\otimes\left(q_{3}-1\right)}\right) I_{q_{4}-1}\left(e_{i_{4}}^{\otimes\left(q_{4}-1\right)}\right)\right) \\
& =\sum_{\beta \in \mathcal{D}_{q}} C_{q, \beta} \prod_{1 \leq j<k \leq 4} \rho\left(i_{j}-i_{k}\right)^{\beta_{j k}},
\end{aligned}
$$

where

$$
C_{q, \beta}=\frac{\prod_{j=1}^{4}\left(q_{j}-1\right) !}{\prod_{1 \leq j<k \leq 4} \beta_{j k} !}
$$

and $\mathcal{D}_{q}$ is the set of nonnegative integers $\beta_{j k}$, satisfying

$$
q_{\ell}-1=\sum_{j \text { or } k=\ell} \beta_{j k}, \quad \text { for } \quad 1 \leq \ell \leq 4 .
$$

On the other hand,

$$
\begin{aligned}
& \mathbb{E}\left(I_{q_{1}-1}\left(e_{i_{1}}^{\otimes\left(q_{1}-1\right)}\right) I_{q_{2}-1}\left(e_{i_{2}}^{\otimes\left(q_{2}-1\right)}\right)\right) \mathbb{E}\left(I_{q_{3}-1}\left(e_{i_{3}}^{\otimes\left(q_{3}-1\right)}\right) I_{q_{4}-1}\left(e_{i_{4}}^{\otimes\left(q_{4}-1\right)}\right)\right) \\
& =\left(q_{1}-1\right) !\left(q_{3}-1\right) ! \rho^{q_{1}-1}\left(i_{1}-i_{2}\right) \rho^{q_{3}-1}\left(i_{3}-i_{4}\right),
\end{aligned}
$$


if $q_{1}=q_{2}$ and $q_{3}=q_{4}$, and zero otherwise. Notice that (4.6) is precisely the term in the sum (4.4) with $\beta_{12}=q_{1}-1, \beta_{34}=q_{3}-1$ and $\beta_{13}=\beta_{14}=\beta_{23}=\beta_{24}=0$. As a consequence, we obtain

$$
\operatorname{Cov}\left(H_{q_{1}-1}\left(X_{i_{1}}\right) H_{q_{2}-1}\left(X_{i_{2}}\right), H_{q_{3}-1}\left(X_{i_{3}}\right) H_{q_{4}-1}\left(X_{i_{4}}\right)\right)=\sum_{\beta \in \mathcal{D}_{q}^{\prime}} C_{q, \beta} \prod_{1 \leq j<k \leq 4} \rho\left(i_{j}-i_{k}\right)^{\beta_{j k}}
$$

where $\mathcal{D}_{q}^{\prime}$ is the set of elements $\left(\beta_{1}, \ldots, \beta_{6}\right)$, where the $\beta_{k}$ 's are nonnegative integers satisfying (4.5) and

$$
\beta_{13}+\beta_{14}+\beta_{23}+\beta_{24} \geq 1 .
$$

Substituting (4.7) into (4.3) yields

$$
\begin{aligned}
\operatorname{Var}\left(\Phi_{n, N}\right)= & \frac{1}{n^{2}} \sum_{i_{1}, i_{2}, i_{3}, i_{4}=1}^{n} \sum_{q_{1}, q_{2}, q_{3}, q_{4}=d}^{N} \sum_{\beta \in \mathcal{D}_{q}^{\prime}} C_{q, \beta} q_{1} q_{3} c_{q_{1}} c_{q_{2}} c_{q_{3}} c_{q_{4}} \\
& \times \rho^{\beta_{12}+1}\left(i_{1}-i_{2}\right) \rho^{\beta_{13}}\left(i_{1}-i_{3}\right) \rho^{\beta_{14}}\left(i_{1}-i_{4}\right) \rho^{\beta_{23}}\left(i_{2}-i_{3}\right) \rho^{\beta_{24}}\left(i_{2}-i_{4}\right) \rho^{\beta_{34}+1}\left(i_{3}-i_{4}\right) .
\end{aligned}
$$

Replacing $\beta_{12}+1$ and $\beta_{34}+1$ by $\beta_{12}$ and $\beta_{34}$, the above equality can be rewritten as

$$
\operatorname{Var}\left(\Phi_{n, N}\right)=\frac{1}{n^{2}} \sum_{i_{1}, i_{2}, i_{3}, i_{4}=1}^{n} \sum_{q_{1}, q_{2}, q_{3}, q_{4}=d}^{N} \sum_{\beta \in \mathcal{E}_{q}} K_{q, \beta} c_{q_{1}} c_{q_{2}} c_{q_{3}} c_{q_{4}} \prod_{1 \leq j<k \leq 4} \rho\left(i_{j}-i_{k}\right)^{\beta_{j k}}
$$

where

$$
K_{q, \beta}=\frac{q_{1} !\left(q_{2}-1\right) ! q_{3} !\left(q_{4}-1\right) !}{\left(\beta_{12}-1\right) ! \beta_{13} ! \beta_{14} ! \beta_{23} ! \beta_{24} !\left(\beta_{34}-1\right) !}
$$

and $\mathcal{E}_{q}$ is the set of nonnegative integers $\beta_{j k}, 1 \leq j<k \leq 4$, satisfying $\beta_{13}+\beta_{14}+\beta_{23}+\beta_{24} \geq 1$, $\beta_{12} \geq 1, \beta_{34} \geq 1$ and

$$
q_{\ell}=\sum_{j \text { or } k=\ell} \beta_{j k}, \quad \text { for } \quad 1 \leq \ell \leq 4
$$

This leads to the estimate

$$
\operatorname{Var}\left(\Phi_{n, N}\right) \leq \sup _{\beta} A_{n, \beta} \sum_{q_{1}, q_{2}, q_{3}, q_{4}=d}^{N} \sum_{\beta \in \mathcal{E}_{q}} K_{q, \beta}\left|c_{q_{1}} c_{q_{2}} c_{q_{3}} c_{q_{4}}\right|,
$$

where

$$
A_{n, \beta}=\frac{1}{n^{2}} \sum_{i_{1}, i_{2}, i_{3}, i_{4}=1}^{n} \prod_{1 \leq j<k \leq 4}\left|\rho\left(i_{j}-i_{k}\right)\right|^{\beta_{j k}}
$$

and the supremum is taken over all sets of nonnegative integers $\beta_{j k}, 1 \leq j<k \leq 4$, satisfying $\beta_{13}+\beta_{14}+\beta_{23}+\beta_{24} \geq 1, \beta_{12} \geq 1, \beta_{34} \geq 1, \beta_{j k} \leq d$ for $1 \leq j<k \leq 4$ and

$$
d \leq \sum_{j \text { or } k=\ell} \beta_{j k}, \quad \text { for } \quad 1 \leq \ell \leq 4 .
$$

To complete the proof we need to show the following claims:

(a) We have

$$
\sum_{q_{1}, q_{2}, q_{3}, q_{4}=d}^{\infty} \sum_{\beta \in \mathcal{E}_{q}} K_{q, \beta}\left|c_{q_{1}} c_{q_{2}} c_{q_{3}} c_{q_{4}}\right|<\infty .
$$

(b) If $d=2$, then $\sup _{\beta} A_{n, \beta}$ is bounded by a constant times the right-hand side of (1.7).

(c) If $d \geq 3$, then $\sup _{\beta} A_{n, \beta}$ is bounded by a constant times the right-hand side of (1.8). 
Proof of (4.8): The main idea here is to identify the sum in (4.8) as the variance of a truncated function composed with a fixed random variable $X_{1}$. From our previous computations it follows that

$$
\begin{aligned}
\sum_{q_{1}, q_{2}, q_{3}, q_{4}=d}^{N} \sum_{\beta \in \mathcal{E}_{q}} K_{q, \beta}\left|c_{q_{1}} c_{q_{2}} c_{q_{3}} c_{q_{4}}\right| & =\sum_{q_{1}, q_{2}, q_{3}, q_{4}=d}^{N} q_{1} q_{3}\left|c_{q_{1}} c_{q_{2}} c_{q_{3}} c_{q_{4}}\right| \\
& \times \operatorname{Cov}\left(H_{q_{1}-1}\left(X_{1}\right) H_{q_{2}-1}\left(X_{1}\right), H_{q_{3}-1}\left(X_{1}\right) H_{q_{4}-1}\left(X_{1}\right)\right) \\
& =\operatorname{Var}\left(A\left(g^{\prime}\right)^{(N)}\left(X_{1}\right) A\left(g_{1}\right)^{(N)}\left(X_{1}\right)\right),
\end{aligned}
$$

where for each integer $N \geq d$, we denote by $A\left(g^{\prime}\right)^{(N)}$ and $A\left(g_{1}\right)^{(N)}$ the truncated expansions of $A\left(g^{\prime}\right)$ and $A\left(g_{1}\right)$, respectively, introduced in (3.2). By Proposition 3.1. $\left(A\left(g^{\prime}\right)^{(N)}\right)^{2}$ and $\left(A\left(g_{1}\right)^{(N)}\right)^{2}$ are convergent in $L^{2}(\mathbb{R}, \gamma)$ to $A\left(g^{\prime}\right)^{2}$ and $A\left(g_{1}\right)^{2}$, respectively. Therefore,

$$
\sum_{q_{1}, q_{2}, q_{3}, q_{4}=d}^{\infty} \sum_{\beta \in \mathcal{E}_{q}} K_{q, \beta}\left|c_{q_{1}} c_{q_{2}} c_{q_{3}} c_{q_{4}}\right|=\operatorname{Var}\left(A\left(g^{\prime}\right)\left(X_{1}\right) A\left(g_{1}\right)\left(X_{1}\right)\right)<\infty .
$$

Proof of (b): We will use ideas from graph theory to show the bound in the first part of Theorem 1. Recall the supremum is taken over all sets of nonnegative integers $\beta_{j k}, 1 \leq j<$ $k \leq 4$, satisfying $\beta_{13}+\beta_{14}+\beta_{23}+\beta_{24} \geq 1, \beta_{12} \geq 1, \beta_{34} \geq 1, \beta_{j k} \leq 2$ for $1 \leq j<k \leq 4$ and

$$
2 \leq \sum_{j \text { or } k=\ell} \beta_{j k}, \quad \text { for } \quad 1 \leq \ell \leq 4 .
$$

The exponents $\beta_{j k}$ induce an unordered simple graph on the set of vertices $V=\{1,2,3,4\}$ by putting an edge between $j$ and $k$ if $\beta_{j k} \neq 0$. There are edges connecting the pairs of vertices $(1,2)$ and $(3,4)$ and condition $\beta_{13}+\beta_{14}+\beta_{23}+\beta_{24} \geq 1$ means that the graph is connected. Without any loss of generality, we can assume that there is an edge between the vertices 2 and 3. Then, condition (4.9) implies that the degree of each vertex is at least two. The worse case is when the number of edges is minimal and the corresponding nonzero coefficients $\beta_{j k}$ are equal to one. So far we have edges in $(1,2),(3,4)$ and $(2,3)$. There must be more edges because each vertex must have at least degree two. There are two possible cases:

(i) $\beta_{14}=1$. In this case we have

$$
A_{n, \beta} \leq \frac{1}{n^{2}} \sum_{i_{1}, i_{2}, i_{3}, i_{4}=1}^{n}\left|\rho\left(i_{1}-i_{2}\right) \rho\left(i_{2}-i_{3}\right) \rho\left(i_{3}-i_{4}\right) \rho\left(i_{1}-i_{4}\right)\right| .
$$

After making the change of variables $i_{1}=i_{1}, k_{1}=i_{1}-i_{2}, k_{2}=i_{2}-i_{3}$ and $k_{3}=i_{3}-i_{4}$ and using the inequality (2.9) with $M=3$ and $v=(1,1,1)$, we obtain

$$
A_{n, \beta} \leq \frac{1}{n} \sum_{\left|k_{i}\right| \leq n, i=1,2,3}\left|\rho\left(k_{1}\right) \rho\left(k_{2}\right) \rho\left(k_{3}\right) \rho\left(k_{1}+k_{2}+k_{3}\right)\right| \leq \frac{C}{n}\left(\sum_{|k| \leq n}|\rho(k)|^{\frac{4}{3}}\right)^{3} .
$$

(ii) Suppose that we add two more edges to the graph formed by the edges $(1,2),(2,3)$ and $(3,4)$. In this case, we obtain

$$
A_{n, \beta} \leq \frac{1}{n^{2}} \sum_{i_{1}, i_{2}, i_{3}, i_{4}=1}^{n}\left|\rho\left(i_{1}-i_{2}\right) \rho\left(i_{2}-i_{3}\right) \rho\left(i_{3}-i_{4}\right) \rho\left(i_{\alpha_{1}}-i_{\beta_{1}}\right) \rho\left(i_{\alpha_{2}}-i_{\beta_{2}}\right)\right| .
$$


Making the change of variables $i_{1}=i_{1}, k_{1}=i_{1}-i_{2}, k_{2}=i_{2}-i_{3}$ and $k_{3}=i_{3}-i_{4}$, we obtain

$$
A_{n, \beta} \leq \frac{1}{n} \sum_{\left|k_{i}\right| \leq n, i=1,2,3}\left|\rho\left(k_{1}\right) \rho\left(k_{2}\right) \rho\left(k_{3}\right) \rho(\mathbf{k} \cdot \mathbf{v}) \rho(\mathbf{k} \cdot \mathbf{w})\right|,
$$

where $\mathbf{v}$ and $\mathbf{w}$ are two linearly independent vectors in $\mathbb{Z}^{3}$ and $\mathbf{k}=\left(k_{1}, k_{2}, k_{3}\right)$. Using (2.11), we obtain

$$
A_{n, \beta} \leq \frac{C}{n} \sum_{|k| \leq n}|\rho(k)|,
$$

which completes the proof of (b).

Proof of $(c)$ : This estimate can be obtained by exactly the same arguments as in the proof of Theorem 4.5 in [17. We omit the details.

Remark 4.1. We can show that both bounds in (1.7) are not comparable. In the particular case $|\rho(k)| \sim|k|^{-\alpha}$ as $|k| \rightarrow \infty$, with $\alpha>\frac{1}{2}$, we obtain:

$$
d_{\mathrm{TV}}\left(Y_{n}, Z\right) \leq \begin{cases}C n^{1-2 \alpha} & \text { if } \frac{1}{2}<\alpha<\frac{2}{3}, \\ C n^{-\frac{\alpha}{2}} & \text { if } \frac{2}{3} \leq \alpha<1, \\ C n^{-\frac{1}{2}}(\log n)^{\frac{1}{2}} & \text { if } \quad \alpha=1, \\ C n^{-\frac{1}{2}} & \text { if } \quad \alpha>1 .\end{cases}
$$

\section{Proof of Theorem 1.2}

Proof. As in the proof of Theorem 1.1, we can assume that $X_{n}=W\left(e_{n}\right)$, where $e_{i} \in \mathfrak{H}, i \geq 0$ are elements in a Hilbert space $\mathfrak{H}$ such that, for each $i, j \geq 0$, we have $\left\langle e_{i}, e_{j}\right\rangle_{\mathfrak{H}}=\rho(i-j)$ and $W=\{W(\phi): \phi \in \mathfrak{H}\}$ is an isonormal Gaussian process.

Consider the sequence $F_{n}:=\frac{1}{\sqrt{n}} \sum_{j=1}^{n} g\left(X_{j}\right)$ introduced in (1.5), where $g \in L^{2}(\mathbb{R}, \gamma)$ has Hermite rank $d=2$ and let $\sigma_{n}^{2}=\mathbb{E}\left(F_{n}^{2}\right)$. Set $Y_{n}=\frac{F_{n}}{\sigma_{n}}$. Taking into account (2.5), we have the representation $Y_{n}=\delta^{2}\left(\frac{1}{\sigma_{n}} v_{n}\right)$, where

$$
v_{n}=\frac{1}{\sqrt{n}} \sum_{j=1}^{n} g_{2}\left(X_{j}\right) e_{j} \otimes e_{j} .
$$

Under condition (1.2), it is well known that as $n \rightarrow \infty, \sigma_{n}^{2} \rightarrow \sigma^{2}$, where $\sigma^{2}$ has been defined in (1.4). As a consequence of Proposition 2.3, we have the estimate

$$
d_{W}\left(Y_{n}, Z\right) \leq C \sqrt{\operatorname{Var}\left(\left\langle D^{2} F_{n}, v_{n}\right\rangle_{\mathfrak{H} \otimes 2}\right)}+C \mathbb{E}\left(\left|\left\langle D F_{n} \otimes D F_{n}, v_{n}\right\rangle_{\mathfrak{H} \otimes 2}\right|\right)
$$

Therefore, we need to estimate the quantities $\operatorname{Var}\left(\left\langle D^{2} F_{n}, v_{n}\right\rangle_{\mathfrak{H}^{\otimes 2}}\right)$ and $\mathbb{E}\left(\left|\left\langle D F_{n} \otimes D F_{n}, v_{n}\right\rangle_{\mathfrak{H}^{\otimes 2}}\right|\right)$.

(i) Estimation of $\operatorname{Var}\left(\left\langle D^{2} F_{n}, v_{n}\right\rangle_{\mathfrak{H} \otimes 2}\right)$. We will follow similar arguments as in the proof of Theorem 1.2, First, we write

$$
\left\langle D^{2} F_{n}, v_{n}\right\rangle_{\mathfrak{H}}^{\otimes 2}=\frac{1}{n} \sum_{i, j=1}^{n} g^{\prime \prime}\left(X_{i}\right) g_{2}\left(X_{j}\right) \rho^{2}(i-j) .
$$

Using a limit argument, we obtain

$$
\left\langle D^{2} F_{n}, V_{n}\right\rangle_{\mathfrak{H}^{\otimes 2}}=\lim _{N \rightarrow \infty} \Phi_{n, N}
$$


where the convergence holds in $L^{1}(\Omega)$ and

$$
\Phi_{n, N}=\frac{1}{n} \sum_{i, j=1}^{n} \sum_{q_{1}, q_{2}=2}^{N} c_{q_{1}} c_{q_{2}} q_{1}\left(q_{1}-1\right) H_{q_{1}-2}\left(X_{i}\right) H_{q_{2}-2}\left(X_{j}\right) \rho^{2}(i-j) .
$$

Therefore, by Fatou's lemma

$$
\operatorname{Var}\left(\left\langle D^{2} F_{n}, v_{n}\right\rangle_{\mathfrak{H}^{\otimes 2}}\right) \leq \liminf _{N \rightarrow \infty} \operatorname{Var}\left(\Phi_{n, N}\right) .
$$

We can write

$$
\begin{aligned}
\operatorname{Var}\left(\Phi_{n, N}\right)= & \frac{1}{n^{2}} \sum_{i_{1}, i_{2}, i_{3}, i_{4}=1}^{n} \sum_{q_{1}, q_{2}, q_{3}, q_{4}=2}^{N} q_{1}\left(q_{1}-1\right) q_{3}\left(q_{3}-1\right) c_{q_{1}} c_{q_{2}} c_{q_{3}} c_{q_{4}} \rho^{2}\left(i_{1}-i_{2}\right) \rho^{2}\left(i_{3}-i_{4}\right) \\
& \times \operatorname{Cov}\left(H_{q_{1}-2}\left(X_{i_{1}}\right) H_{q_{2}-2}\left(X_{i_{2}}\right), H_{q_{3}-2}\left(X_{i_{3}}\right) H_{q_{4}-2}\left(X_{i_{4}}\right)\right) .
\end{aligned}
$$

With a very similar calculation as in the proof of Theorem 1.1, we have

$$
\operatorname{Cov}\left(H_{q_{1}-1}\left(X_{i_{1}}\right) H_{q_{2}-1}\left(X_{i_{2}}\right), H_{q_{3}-1}\left(X_{i_{3}}\right) H_{q_{4}-1}\left(X_{i_{4}}\right)\right)=\sum_{\beta \in \mathcal{D}_{q}^{\prime}} C_{q, \beta} \prod_{1 \leq j<k \leq 4} \rho\left(i_{j}-i_{k}\right)^{\beta_{j k}}
$$

where $\mathcal{D}_{q}^{\prime}$ is the set of nonnegative integers $\beta_{j k}, 1 \leq j<k \leq 4$, satisfying

$$
q_{\ell}-2=\sum_{j \text { or } k=\ell} \beta_{j k}, \quad \text { for } \quad 1 \leq \ell \leq 4
$$

and

$$
\beta_{13}+\beta_{14}+\beta_{23}+\beta_{24} \geq 1 .
$$

Substituting (5.4) into (5.3) yields

$$
\begin{aligned}
\operatorname{Var}\left(\Phi_{n, N}\right)= & \frac{1}{n^{2}} \sum_{i_{1}, i_{2}, i_{3}, i_{4}=1}^{n} \sum_{q_{1}, q_{2}, q_{3}, q_{4}=2}^{N} \sum_{\beta \in \mathcal{D}_{q}^{\prime}} C_{q, \beta} q_{1}\left(q_{1}-1\right) q_{3}\left(q_{3}-1\right) c_{q_{1}} c_{q_{2}} c_{q_{3}} c_{q_{4}} \\
& \times \rho^{\beta_{12}+2}\left(i_{1}-i_{2}\right) \rho^{\beta_{13}}\left(i_{1}-i_{3}\right) \rho^{\beta_{14}}\left(i_{1}-i_{4}\right) \rho^{\beta_{23}}\left(i_{2}-i_{3}\right) \rho^{\beta_{24}}\left(i_{2}-i_{4}\right) \rho^{\beta_{34}+2}\left(i_{3}-i_{4}\right) .
\end{aligned}
$$

Replacing $\beta_{12}+2$ and $\beta_{34}+2$ by $\beta_{12}$ and $\beta_{34}$, the above equality can be rewritten as

$$
\operatorname{Var}\left(\Phi_{n, N}\right)=\frac{1}{n^{2}} \sum_{i_{1}, i_{2}, i_{3}, i_{4}=1}^{n} \sum_{q_{1}, q_{2}, q_{3}, q_{4}=2}^{N} \sum_{\beta \in \mathcal{E}_{q}} K_{q, \beta} c_{q_{1}} c_{q_{2}} c_{q_{3}} c_{q_{4}} \prod_{1 \leq j<k \leq 4} \rho\left(i_{j}-i_{k}\right)^{\beta_{j k}},
$$

where

$$
K_{q, \beta}=\frac{q_{1} !\left(q_{2}-2\right) ! q_{3} !\left(q_{4}-2\right) !}{\left(\beta_{12}-2\right) ! \beta_{13} ! \beta_{14} ! \beta_{23} ! \beta_{24} !\left(\beta_{34}-2\right) !}
$$

and $\mathcal{E}_{q}$ is the set of nonnegative integers $\beta_{j k}, 1 \leq j<k \leq 4$, satisfying $\beta_{13}+\beta_{14}+\beta_{23}+\beta_{24} \geq 1$, $\beta_{12} \geq 2, \beta_{34} \geq 2$ and

$$
q_{\ell}=\sum_{j \text { or } k=\ell} \beta_{j k}, \quad \text { for } \quad 1 \leq \ell \leq 4
$$

We can write

$$
\operatorname{Var}\left(\Phi_{n, N}\right) \leq \sup _{\beta} A_{n, \beta} \sum_{q_{1}, q_{2}, q_{3}, q_{4}=2}^{N} \sum_{\beta \in \mathcal{E}_{q}} K_{q, \beta}\left|c_{q_{1}} c_{q_{2}} c_{q_{3}} c_{q_{4}}\right|
$$


where

$$
A_{n, \beta}=\frac{1}{n^{2}} \sum_{i_{1}, i_{2}, i_{3}, i_{4}=1}^{n} \prod_{1 \leq j<k \leq 4}\left|\rho\left(i_{j}-i_{k}\right)\right|^{\beta_{j k}}
$$

and the supremum is taken over all sets of nonnegative integers $\beta_{j k}, 1 \leq j<k \leq 4$, satisfying $\beta_{13}+\beta_{14}+\beta_{23}+\beta_{24} \geq 1, \beta_{12} \geq 2, \beta_{34} \geq 2$, for $1 \leq j<k \leq 4$ and

$$
2 \leq \sum_{j \text { or } k=\ell} \beta_{j k}, \quad \text { for } \quad 1 \leq \ell \leq 4
$$

Then, in this case we have

$$
A_{n, \beta} \leq \frac{1}{n^{2}} \sum_{i_{1}, i_{2}, i_{3}, i_{4}=1}^{n}\left|\rho\left(i_{1}-i_{2}\right)^{2} \rho\left(i_{\alpha_{1}}-i_{\alpha_{2}}\right) \rho\left(i_{3}-i_{4}\right)^{2}\right|
$$

where $\alpha_{1} \in\{1,2\}$ and $\alpha_{2} \in\{3,4\}$. After making the change $i_{1}=i_{1}, k_{1}=i_{1}-i_{2}, k_{2}=i_{\alpha_{1}}-i_{\alpha_{2}}$ and $k_{3}=i_{3}-i_{4}$, we obtain

$$
A_{n, \beta} \leq \frac{1}{n} \sum_{\left|k_{i}\right| \leq n, i=1,2,3}\left|\rho\left(k_{1}\right)^{2} \rho\left(k_{2}\right) \rho\left(k_{3}\right)^{2}\right| \leq \frac{C}{n} \sum_{|k| \leq n}|\rho(k)| .
$$

Now, it is left to show that

$$
\sum_{q_{1}, q_{2}, q_{3}, q_{4}=2}^{N} \sum_{\beta \in \mathcal{E}_{q}} K_{q, \beta}\left|c_{q_{1}} c_{q_{2}} c_{q_{3}} c_{q_{4}}\right|<\infty
$$

We have

$$
\begin{aligned}
& \sum_{q_{1}, q_{2}, q_{3}, q_{4}=2}^{N} \sum_{\beta \in \mathcal{E}_{q}} K_{q, \beta}\left|c_{q_{1}} c_{q_{2}} c_{q_{3}} c_{q_{4}}\right|=\sum_{q_{1}, q_{2}, q_{3}, q_{4}=2}^{N} q_{1}\left(q_{1}-1\right) q_{3}\left(q_{3}-1\right)\left|c_{q_{1}} c_{q_{2}} c_{q_{3}} c_{q_{4}}\right| \\
\times & \mathbb{E}\left(H_{q_{1}-2}\left(X_{1}\right) H_{q_{2}-2}\left(X_{1}\right) H_{q_{3}-2}\left(X_{1}\right) H_{q_{4}-2}\left(X_{1}\right)\right) \\
= & \mathbb{E}\left(\left(A\left(g^{\prime \prime}\right)^{(N)}\right)^{2}\left(A\left(g_{2}\right)^{(N)}\right)^{2}\right) .
\end{aligned}
$$

By Hölder's inequality, we obtain

$$
\sum_{q_{1}, q_{2}, q_{3}, q_{4}=2}^{N} \sum_{\beta \in \mathcal{E}_{q}} K_{q, \beta}\left|c_{q_{1}} c_{q_{2}} c_{q_{3}} c_{q_{4}}\right| \leq\left\|A\left(g^{\prime \prime}\right)^{(N)}\right\|_{L^{4}(\mathbb{R}, \gamma)}^{1 / 2}\left\|A\left(g_{2}\right)^{(N)}\right\|_{L^{4}(\mathbb{R}, \gamma)}^{1 / 2} .
$$

From the hypothesis and the Proposition 3.1. $\left(A\left(g^{\prime \prime}\right)^{(N)}\right)^{2}$ and $\left(A\left(g_{2}\right)^{(N)}\right)^{2}$ converge to $A\left(g^{\prime \prime}\right)^{2}$ and $A\left(g_{2}\right)^{2}$ in $L^{2}(\mathbb{R}, \gamma)$ respectively. Hence, (5.6) holds.

(ii) Estimation of $\mathbb{E}\left(\left|\left\langle D F_{n} \otimes D F_{n}, v_{n}\right\rangle_{\mathfrak{H}^{\otimes 2}}\right|\right)$. We can write

$$
\left\langle D F_{n} \otimes D F_{n}, v_{n}\right\rangle_{\mathfrak{H}^{\otimes 2}}=n^{-\frac{3}{2}} \sum_{i, j, k=1}^{n} g^{\prime}\left(X_{i}\right) g^{\prime}\left(X_{j}\right) g_{2}\left(X_{k}\right) \rho(i-k) \rho(j-k) .
$$

We have, in the $L^{1}(\Omega)$ sense,

$$
\left\langle D F_{n}, u_{n}\right\rangle_{\mathfrak{H}}=\lim _{N \rightarrow \infty} \Psi_{n, N}
$$


where

$$
\Psi_{n, N}=n^{-\frac{3}{2}} \sum_{i, j, k=1}^{n} \sum_{q_{1}, q_{2}, q_{3}=2}^{N} c_{q_{1}} c_{q_{2}} c_{q_{3}} q_{1} q_{2} H_{q_{1}-1}\left(X_{i}\right) H_{q_{2}-1}\left(X_{j}\right) H_{q_{3}-2}\left(X_{k}\right) \rho(i-k) \rho(j-k) .
$$

Therefore, by Fatou's lemma

$$
\mathbb{E}\left(\langle D F \otimes D F, v\rangle_{\mathfrak{H}^{\otimes 2}}^{2}\right) \leq \liminf _{N \rightarrow \infty} \mathbb{E}\left(\Psi_{n, N}^{2}\right) .
$$

We can write

$$
\begin{aligned}
\mathbb{E}\left(\Psi_{n, N}^{2}\right)=n^{-3} & \sum_{i_{1}, \ldots, i_{6}=1}^{n} \sum_{q_{1}, \ldots, q_{6}=2}^{N}\left(\prod_{i=1}^{6} c_{q_{i}}\right) q_{1} q_{2} q_{4} q_{5} \\
& \times \mathbb{E}\left(H_{q_{1}-1}\left(X_{i_{1}}\right) H_{q_{2}-1}\left(X_{i_{2}}\right) H_{q_{3}-2}\left(X_{i_{3}}\right) H_{q_{4}-1}\left(X_{i_{4}}\right) H_{q_{5}-1}\left(X_{i_{5}}\right) H_{q_{6}-2}\left(X_{i_{6}}\right)\right) \\
& \times \rho\left(i_{1}-i_{3}\right) \rho\left(i_{2}-i_{3}\right) \rho\left(i_{4}-i_{6}\right) \rho\left(i_{5}-i_{6}\right) .
\end{aligned}
$$

Using Lemma 3.1, we obtain

$$
\begin{aligned}
& \mathbb{E}\left(H_{q_{1}-1}\left(X_{i_{1}}\right) H_{q_{2}-1}\left(X_{i_{2}}\right) H_{q_{3}-2}\left(X_{i_{3}}\right) H_{q_{4}-1}\left(X_{i_{4}}\right) H_{q_{5}-1}\left(X_{i_{5}}\right) H_{q_{6}-2}\left(X_{i_{6}}\right)\right) \\
& =\sum_{\beta \in \mathcal{D}_{q}} C_{q, \beta} \prod_{1 \leq j<k \leq 6} \rho\left(i_{j}-i_{k}\right)^{\beta_{j k}}
\end{aligned}
$$

where

$$
C_{q, \beta}=\frac{\left(q_{3}-2\right) !\left(q_{6}-2\right) ! \prod_{j=1,2,4,5}^{4}\left(q_{j}-1\right) !}{\prod_{1 \leq j<k \leq 6} \beta_{j k} !}
$$

and $\mathcal{D}_{q}$ is the set of nonnegative integers $\beta_{j k}, 1 \leq j<k \leq 6$, satisfying

$$
\begin{aligned}
q_{\ell}-1 & =\sum_{j \text { or } k=\ell} \beta_{j k}, \quad \text { for } \quad \ell=1,2,4,5, \\
q_{3}-2 & =\sum_{j \text { or } k=3} \beta_{j k}, \\
q_{6}-2 & =\sum_{j \text { or } k=6} \beta_{j k} .
\end{aligned}
$$

Replacing (5.8) into (5.7) yields

$$
\begin{aligned}
\mathbb{E}\left(\Psi_{n, N}^{2}\right)=n^{-3} & \sum_{i_{1}, \ldots, i_{6}=1}^{n} \sum_{q_{1}, \ldots, q_{6}=2}^{N} \sum_{\beta \in \mathcal{D}_{q}} C_{q, \beta}\left(\prod_{i=1}^{6} c_{q_{i}}\right) q_{1} q_{2} q_{4} q_{5} \\
& \times \rho\left(i_{1}-i_{3}\right) \rho\left(i_{2}-i_{3}\right) \rho\left(i_{4}-i_{6}\right) \rho\left(i_{5}-i_{6}\right) \prod_{j, k=1, j<k}^{6} \rho\left(i_{j}-i_{k}\right)^{\beta_{j k}} .
\end{aligned}
$$

Substituting $\beta_{13}+1, \beta_{23}+1, \beta_{46}+1$ and $\beta_{56}+1$ by $\beta_{13}, \beta_{23}, \beta_{46}$ and $\beta_{56}$, respectively, we can write

$$
\mathbb{E}\left(\Psi_{n, N}^{2}\right)=n^{-3} \sum_{i_{1}, \ldots, i_{6}=1}^{n} \sum_{q_{1}, \ldots, q_{6}=2}^{N} \sum_{\beta \in \mathcal{E}_{q}} K_{q, \beta}\left(\prod_{i=1}^{6} c_{q_{i}}\right) q_{1} q_{2} q_{4} q_{5} \prod_{j, k=1, j<k}^{6} \rho\left(i_{j}-i_{k}\right)^{\beta_{j k}},
$$


where

$$
K_{q, \beta}=\frac{\beta_{13} \beta_{23} \beta_{46} \beta_{56}\left(q_{3}-2\right) !\left(q_{6}-2\right) ! \prod_{j=1,2,4,5}^{4}\left(q_{j}-1\right) !}{\prod_{j, k=1, j<k}^{6} \beta_{j k} !}
$$

and $\mathcal{E}_{q}$ is the set of nonnegative integers $\beta_{j k}, 1 \leq j<k \leq 6$, satisfying

$$
q_{\ell}=\sum_{j \text { or } k=\ell} \beta_{j k}, \quad \text { for } \quad \ell=1, \ldots, 6
$$

Hence

$$
\mathbb{E}\left(\Psi_{n, N}^{2}\right) \leq \sup _{\beta} A_{n, \beta} \sum_{q_{1}, \ldots, q_{6}=2}^{N} \sum_{\beta \in \mathcal{E}_{q}} K_{q, \beta}\left(\prod_{i=1}^{6}\left|c_{q_{i}}\right|\right) q_{1} q_{2} q_{4} q_{5}
$$

where

$$
A_{n, \beta}=n^{-3} \sum_{i_{1}, \ldots, i_{6}=1}^{n} \prod_{1 \leq j<k \leq 6}\left|\rho\left(i_{j}-i_{k}\right)\right|^{\beta_{j k}}
$$

and the supremum is taken over all sets of nonnegative integers $\beta_{j k}, j, k=1, \ldots, 6, j<k$, satisfying $\beta_{13} \geq 1, \beta_{23} \geq 1, \beta_{46} \geq 1, \beta_{56} \geq 1$ and

$$
2 \leq \sum_{j \text { or } k=\ell} \beta_{j k}, \text { for } \ell=1, \ldots, 6 .
$$

As in the proof of Theorem 1.1, we can show that

$$
\sum_{q_{1}, \ldots, q_{6}=2}^{\infty} \sum_{\beta \in \mathcal{E}_{q}} K_{q, \beta}\left(\prod_{i=1}^{6}\left|c_{q_{i}}\right|\right) q_{1} q_{2} q_{4} q_{5}<\infty
$$

In fact,

$$
\begin{aligned}
& \sum_{q_{1}, \ldots, q_{6}=2}^{N} \sum_{\beta \in \mathcal{E}_{q}} K_{q, \beta}\left(\prod_{i=1}^{6}\left|c_{q_{i}}\right|\right) q_{1} q_{2} q_{4} q_{5}=\sum_{q_{1}, \ldots, q_{6}=2}^{N}\left(\prod_{i=1}^{6}\left|c_{q_{i}}\right|\right) q_{1} q_{2} q_{4} q_{5} \\
\times & \mathbb{E}\left[H_{q_{1}-1}\left(X_{1}\right) H_{q_{2}-1}\left(X_{1}\right) H_{q_{3}-2}\left(X_{1}\right) H_{q_{4}-1}\left(X_{1}\right) H_{q_{5}-1}\left(X_{1}\right) H_{q_{6}-2}\left(X_{1}\right)\right] \\
= & \mathbb{E}\left[\left(A\left(g^{\prime}\right)^{(N)}\right)^{4}\left(X_{1}\right)\left(A\left(g_{2}\right)^{(N)}\right)^{2}\left(X_{1}\right)\right],
\end{aligned}
$$

where, as before, $A\left(g^{\prime}\right)^{(N)}$ and $A\left(g_{2}\right)^{(N)}$ are the truncated expansions of $A\left(g^{\prime}\right)$ and $A\left(g_{2}\right)$, respectively. By Hölder's inequality, we can write

$$
\sum_{q_{1}, \ldots, q_{6}=2}^{N} \sum_{\beta \in \mathcal{E}_{q}} K_{q, \beta}\left(\prod_{i=1}^{6}\left|c_{q_{i}}\right|\right) q_{1} q_{2} q_{4} q_{5} \leq\left\|A\left(g^{\prime}\right)^{(N)}\right\|_{L^{6}(\mathbb{R}, \gamma)}^{\frac{2}{3}}\left\|A\left(g_{2}\right)^{(N)}\right\|_{L^{6}(\mathbb{R}, \gamma)}^{\frac{1}{3}} .
$$

From our hypothesis and in view of Proposition 3.1. $\left(A\left(g^{\prime}\right)^{(N)}\right)^{3}$ and $\left(A\left(g_{2}\right)^{(N)}\right)^{3}$ converge in $L^{2}(\mathbb{R}, \gamma)$ to $A\left(g^{\prime}\right)$ and $A\left(g_{2}\right)$, respectively. Thus, (5.11) holds true.

To complete the proof, it remains to show that,

$$
\sup _{\beta} A_{n, \beta} \leq C n^{-1}\left(\sum_{|k| \leq n}|\rho(k)|^{\frac{3}{2}}\right)^{4} .
$$

As in the proof of Theorem 1.1, in order to show this estimate we will make use of some ideas from graph theory. The exponents $\beta_{j k}$ induce an unordered simple graph on the set of vertices $V=\{1,2,3,4,5,6\}$ by putting an edge between $j$ and $k$ whenever $\beta_{j k} \neq 0$. Because 
$\beta_{13} \geq 1, \beta_{23} \geq 1, \beta_{46} \geq 1$ and $\beta_{56} \geq 1$, there are edges connecting the pairs of vertices $(1,3)$, $(2,3),(4,6)$ and $(5,6)$. Condition (5.10) means that the degree of each vertex is at least 2 . Then we consider two cases, depending whether graph is connected or not.

Case 1: Suppose that the graph is not connected. This implies that $\beta_{12} \geq 1, \beta_{45} \geq 1$ and there is no edge between the sets $V_{1}=\{1,2,3\}$ and $V_{2}=\{4,5,6\}$. The worse case is when $\beta_{12}=\beta_{13}=\beta_{23}=\beta_{45}=\beta_{46}=\beta_{56}=1$ and all the other exponents are zero. In this case we have the estimate

$$
A_{n, \beta} \leq n^{-1}\left(\sum_{\left|k_{1}\right|,\left|k_{2}\right| \leq n}\left|\rho\left(k_{1}\right) \rho\left(k_{2}\right) \rho\left(k_{1}-k_{2}\right)\right|\right)^{2} .
$$

Using (2.9), we obtain

$$
A_{n, \beta} \leq C n^{-1}\left(\sum_{|k| \leq n}|\rho(k)|^{\frac{3}{2}}\right)^{4}
$$

Case 2: Suppose that the graph is connected. This means that there is an edge connecting the sets $V_{1}$ and $V_{2}$. Suppose that $\beta_{\alpha_{0} \delta_{0}} \geq 1$, where $\alpha_{0} \in\{1,2,3\}$ and $\delta_{0} \in\{4,5,6\}$. We have then 5 nonzero coefficients $\beta$ : $\beta_{13}, \beta_{23}, \beta_{46}, \beta_{56}$ and $\beta_{\alpha_{0} \delta_{0}}$. Because all the edges have at least degree 2 , there must be at least two more nonzero coefficients $\beta$. Let us denote them by $\beta_{\alpha_{1} \delta_{1}}$ and $\beta_{\alpha_{2} \delta_{2}}$.

Then, the worse case will be when $\beta_{13}=\beta_{23}=\beta_{46}=\beta_{56}=\beta_{\alpha_{0} \delta_{0}}=\beta_{\alpha_{1} \delta_{1}}=\beta_{\alpha_{2} \delta_{2}}=1$ and all the other coefficients are zero. Consider the change of variables $i_{1}-i_{3}=k_{1}, i_{2}-i_{3}=k_{2}$, $i_{4}-i_{6}=k_{3}, i_{5}-i_{6}=k_{4}, i_{\alpha_{0}}-i_{\delta_{0}}=k_{5}$. Then, $i_{\alpha_{1}}-i_{\delta_{1}}=\mathbf{k} \cdot \mathbf{v}$ and $i_{\alpha_{2}}-i_{\delta_{2}}=\mathbf{k} \cdot \mathbf{w}$, where $\mathbf{k}=\left(k_{1}, \ldots, k_{5}\right)$ and $\mathbf{v}, \mathbf{w}$ are 5-dimensional linearly independent vectors whose components are 0,1 or -1 . Then, we can write, using (2.11) and Hölder's inequality,

$$
\begin{aligned}
A_{n, \beta} & \leq n^{-2} \sum_{\left|k_{i}\right| \leq n, 2 \leq i \leq 5} \prod_{i=2}^{5}\left|\rho\left(k_{i}\right)\right||\rho(\mathbf{k} \cdot \mathbf{v}) \rho(\mathbf{k} \cdot \mathbf{w})| \leq C n^{-2}\left(\sum_{|k| \leq n}|\rho(k)|\right)^{3} \\
& \leq C n^{-1}\left(\sum_{|k| \leq n}|\rho(k)|^{\frac{3}{2}}\right)^{4}
\end{aligned}
$$

Remark 5.1. In the case $g(x)=x^{2}-1$, the term $\operatorname{Var}\left(\left\langle D^{2} F_{n}, v_{n}\right\rangle_{\mathfrak{H}^{\otimes 2}}\right)$ is zero because $\left\langle D^{2} F_{n}, v_{n}\right\rangle_{\mathfrak{H} \otimes 2}$ is deterministic, and for the second term we get the estimate (1.11).

Remark 5.2. We can show that both bounds in (1.10) are not comparable. In the particular case $|\rho(k)| \sim|k|^{-\alpha}$ as $|k| \rightarrow \infty$, with $\alpha>\frac{1}{2}$, we obtain:

$$
d_{\mathrm{W}}\left(Y_{n}, Z\right) \leq \begin{cases}C n^{\frac{3}{2}-3 \alpha} & \text { if } \frac{1}{2}<\alpha \leq \frac{3}{5}, \\ C n^{-\frac{\alpha}{2}} & \text { if } \frac{3}{5}<\alpha \leq 1, \\ C n^{-\frac{1}{2}}(\log n)^{\frac{1}{2}} & \text { if } \alpha=1, \\ C n^{-\frac{1}{2}} & \text { if } \quad \alpha>1 .\end{cases}
$$




\section{Proof of Theorem 1.3}

Proof. With the notation used in the proof of Theorem 1.1 and using Proposition 2.2, we can write

$$
\begin{aligned}
d_{T V}\left(Y_{n}, Z\right) \leq & (8+\sqrt{32 \pi}) \operatorname{Var}\left(\left\langle D Y_{n}, u_{n} / \sigma_{n}\right\rangle_{\mathfrak{H}}\right)+\sqrt{2 \pi}\left|\mathbb{E}\left(Y_{n}^{3}\right)\right|+\sqrt{32 \pi} \mathbb{E}\left(\left|D_{u_{n} / \sigma_{n}}^{2} Y_{n}\right|^{2}\right) \\
& +4 \pi \mathbb{E}\left(\left|D_{u_{n} / \sigma_{n}}^{3} Y_{n}\right|\right) \\
\leq C & \left(\operatorname{Var}\left(\left\langle D F_{n}, u_{n}\right\rangle_{\mathfrak{H}}+\left|\mathbb{E}\left(F_{n}^{3}\right)\right|+\mathbb{E}\left(\left|D_{u_{n}}^{2} F_{n}\right|^{2}\right)+\sqrt{\mathbb{E}\left(\left|D_{u_{n}}^{3} F_{n}\right|^{2}\right)}\right)\right.
\end{aligned}
$$

Now, we want to estimate each of these terms separately.

Step 1. From Theorem 1.1 we know that

$$
\operatorname{Var}\left(\left\langle D F_{n}, u_{n}\right\rangle_{\mathfrak{H}} \leq C n^{-1} \sum_{|k| \leq n}|\rho(k)|+C n^{-1}\left(\sum_{|k| \leq n}|\rho(k)|^{\frac{4}{3}}\right)^{3} .\right.
$$

Step 2. We claim that

$$
\left|\mathbb{E}\left(F_{n}^{3}\right)\right| \leq \frac{C}{\sqrt{n}}\left(\sum_{|k| \leq n}|\rho(k)|^{\frac{3}{2}}\right)^{2}
$$

We can write

$$
F_{n}^{3}=\frac{1}{n^{3 / 2}} \sum_{i, j, k=1}^{n} g\left(X_{i}\right) g\left(X_{j}\right) g\left(X_{k}\right)
$$

Truncating the Wiener chaos expansion of the random variables $g\left(X_{i}\right)$, as in the proof of Theorem 1.1, we obtain

$$
F_{n}^{3}=\lim _{N \rightarrow \infty} \Psi_{n, N}^{3}:=\lim _{N \rightarrow \infty} \frac{1}{\sqrt{n}} \sum_{i=1}^{n} \sum_{q=2}^{N} c_{q} H_{q}\left(X_{i}\right),
$$

where the convergence holds in $L^{2}(\Omega)$ due to Proposition 3.1 because $g \in L^{6}(\mathbb{R}, \gamma)$. Therefore,

$$
\mathbb{E}\left(F_{n}^{3}\right)=\lim _{N \rightarrow \infty} \mathbb{E}\left(\Psi_{n, N}^{3}\right) .
$$

We can write

$$
\begin{aligned}
\mathbb{E}\left(\Psi_{n, N}^{3}\right)= & \frac{1}{n^{3 / 2}} \sum_{i_{1}, i_{2}, i_{3}=1}^{n} \sum_{q_{1}, q_{2}, q_{3}=2}^{N} c_{q_{1}} c_{q_{2}} c_{q_{3}} \mathbb{E}\left(H_{q_{1}}\left(X_{i_{1}}\right) H_{q_{2}}\left(X_{i_{2}}\right) H_{q_{3}}\left(X_{i_{3}}\right)\right) \\
& =\frac{1}{n^{3 / 2}} \sum_{i_{1}, i_{2}, i_{3}=1}^{n} \sum_{q_{1}, q_{2}, q_{3}=2}^{N} c_{q_{1}} c_{q_{2}} c_{q_{3}} \mathbb{E}\left(I_{q_{1}}\left(e_{i_{1}}^{\otimes q_{1}}\right) I_{q_{2}}\left(e_{i_{2}}^{\otimes q_{2}}\right) I_{q_{3}}\left(e_{i_{3}}^{\otimes q_{3}}\right)\right) .
\end{aligned}
$$

Using Lemma 3.1, we obtain

$$
\mathbb{E}\left(I_{q_{1}}\left(e_{i_{1}}^{\otimes q_{1}}\right) I_{q_{2}}\left(e_{i_{2}}^{\otimes q_{2}}\right) I_{q_{3}}\left(e_{i_{3}}^{\otimes q_{3}}\right)\right)=\sum_{\beta \in \mathcal{D}_{q}} C_{q, \beta} \prod_{1 \leq j<k \leq 3} \rho\left(i_{j}-i_{k}\right)^{\beta_{j k}},
$$

where

$$
C_{q, \beta}=\frac{\prod_{j=1}^{3} q_{j} !}{\prod_{1 \leq j<k \leq 3} \beta_{j k} !}
$$


and $\mathcal{D}_{q}$ is the set of nonnegative integers $\beta_{j k}, 1 \leq j<k \leq 3$, satisfying

$$
q_{\ell}=\sum_{j \text { or } k=\ell} \beta_{j k}, \quad \text { for } \quad 1 \leq \ell \leq 3 .
$$

Then,

$$
\left|\mathbb{E}\left(\Psi_{n, N}^{3}\right)\right| \leq \sup _{\beta} A_{n, \beta} \sum_{q_{1}, q_{2}, q_{3}=2}^{N} \sum_{\beta \in \mathcal{E}_{q}} C_{q, \beta}\left|c_{q_{1}} c_{q_{2}} c_{q_{3}}\right|
$$

where

$$
A_{n, \beta}=\frac{1}{n^{3 / 2}} \sum_{i_{1}, i_{2}, i_{3}=1}^{n} \prod_{1 \leq j<k \leq 3}\left|\rho\left(i_{j}-i_{k}\right)\right|^{\beta_{j k}},
$$

and the supremum is taken over all sets of nonnegative integers $\beta_{j k}, 1 \leq j<k \leq 3$, satisfying $\beta_{j k} \leq 2$ for $1 \leq j<k \leq 3$ and

$$
2 \leq \sum_{j \text { or } k=\ell} \beta_{j k}, \quad \text { for } \quad 1 \leq \ell \leq 3 .
$$

It is easy to see that to satisfy the above conditions, $\beta_{j k} \geq 1$ for all $1 \leq j<k \leq 3$. Hence, we have

$$
A_{n, \beta} \leq \frac{1}{n^{3 / 2}} \sum_{i_{1}, i_{2}, i_{3}=1}^{n}\left|\rho\left(i_{1}-i_{2}\right) \rho\left(i_{1}-i_{3}\right) \rho\left(i_{2}-i_{3}\right)\right| .
$$

After making the change of variables $i_{1}=i_{1}, k_{1}=i_{1}-i_{2}, k_{2}=i_{1}-i_{3}$ and using the inequality (2.9) with $M=2$ and $v=(-1,1)$, we obtain

$$
A_{n, \beta} \leq \frac{1}{n^{1 / 2}} \sum_{\left|k_{1}\right|,\left|k_{2}\right| \leq n}\left|\rho\left(k_{1}\right) \rho\left(k_{2}\right) \rho\left(k_{2}-k_{1}\right)\right| \leq \frac{C}{\sqrt{n}}\left(\sum_{|k| \leq n}|\rho(k)|^{\frac{3}{2}}\right)^{2} .
$$

To complete the proof of (6.3), we need to show that:

$$
\sum_{q_{1}, q_{2}, q_{3}=2}^{\infty} \sum_{\beta \in \mathcal{D}_{q}} C_{q, \beta}\left|c_{q_{1}} c_{q_{2}} c_{q_{3}}\right|<\infty .
$$

In fact,

$$
\left.\lim _{N \rightarrow \infty} \sum_{q_{1}, q_{2}, q_{3}=2}^{N} \sum_{\beta \in \mathcal{D}_{q}} C_{q, \beta}\left|c_{q_{1}} c_{q_{2}} c_{q_{3}}\right|=\lim _{N \rightarrow \infty} \mathbb{E}\left(A(g)^{N}\right)^{3}\right)=\mathbb{E}\left((A(g))^{3}\right)<\infty,
$$

taking into account Proposition 3.1 and the fact that $A(g) \in L^{6}(\mathbb{R}, \gamma)$.

Step 3. We proceed now with the estimation of $\mathbb{E}\left(\left|D_{u_{n}}^{2} F_{n}\right|^{2}\right)$. We can write

$$
D_{u_{n}} F_{n}=\left\langle D F_{n}, u_{n}\right\rangle_{\mathfrak{H}}=\frac{1}{n} \sum_{i, j=1}^{n} g^{\prime}\left(X_{i}\right) g_{1}\left(X_{j}\right) \rho(i-j)
$$

and

$$
D\left(\left\langle D F_{n}, u_{n}\right\rangle_{\mathfrak{H}}\right)=\frac{1}{n} \sum_{i, j=1}^{n}\left(g^{\prime \prime}\left(X_{i}\right) g_{1}\left(X_{j}\right) e_{i}+g^{\prime}\left(X_{i}\right) g_{1}^{\prime}\left(X_{j}\right) e_{j}\right) \rho(i-j) .
$$


Therefore,

$$
\begin{aligned}
D_{u_{n}}^{2} F_{n} & =\left\langle u_{n}, D\left(\left\langle D F_{n}, u_{n}\right\rangle_{\mathfrak{H}}\right)\right\rangle_{\mathfrak{H}} \\
6.7) & =\frac{1}{n^{3 / 2}} \sum_{i, j, k=1}^{n}\left(g^{\prime \prime}\left(X_{i}\right) g_{1}\left(X_{j}\right) g_{1}\left(X_{k}\right) \rho(i-k)+g^{\prime}\left(X_{i}\right) g_{1}^{\prime}\left(X_{j}\right) g_{1}\left(X_{k}\right) \rho(j-k)\right) \rho(i-j) .
\end{aligned}
$$

Because the random variables $g^{\prime \prime}\left(X_{i}\right), g_{1}\left(X_{j}\right), g_{1}\left(X_{k}\right), g^{\prime}\left(X_{i}\right)$ and $g_{1}^{\prime}\left(X_{j}\right)$ appearing in the above expression belong to $L^{2}(\Omega)$, their truncated Wiener chaos expansions convergence in $L^{2}(\Omega)$, and, as a consequence, $D_{u_{n}}^{2} F_{n}=\lim _{N \rightarrow \infty} \Phi_{n, N}$ in probability, where

$$
\begin{aligned}
\Phi_{n, N}= & \frac{1}{n^{3 / 2}} \sum_{i_{1}, i_{2}, i_{3}=1}^{n} \sum_{q_{1}, q_{2}, q_{3}=2}^{N} c_{q_{1}} c_{q_{2}} c_{q_{3}} q_{1}\left(q_{1}-1\right) H_{q_{1}-2}\left(X_{i_{1}}\right) H_{q_{2}-1}\left(X_{i_{2}}\right) H_{q_{3}-1}\left(X_{i_{3}}\right) \\
& \times \rho\left(i_{1}-i_{2}\right) \rho\left(i_{1}-i_{3}\right) \\
& +c_{q_{1}} c_{q_{2}} c_{q_{3}} q_{1}\left(q_{2}-1\right) H_{q_{1}-1}\left(X_{i_{1}}\right) H_{q_{2}-2}\left(X_{i_{2}}\right) H_{q_{3}-1}\left(X_{i_{3}}\right) \rho\left(i_{1}-i_{2}\right) \rho\left(i_{2}-i_{3}\right) .
\end{aligned}
$$

Making the change of variables $\left(q_{1}, q_{2}\right) \rightarrow\left(q_{2}, q_{1}\right)$ and $\left(i_{1}, i_{2}\right) \rightarrow\left(i_{2}, i_{1}\right)$ in the second sum allows us to put the two terms together, and we obtain

$$
\begin{aligned}
\Phi_{n, N}= & \frac{1}{n^{3 / 2}} \sum_{i_{1}, i_{2}, i_{3}=1}^{n} \sum_{q_{1}, q_{2}, q_{3}=2}^{N} c_{q_{1}} c_{q_{2}} c_{q_{3}}\left(q_{1}+q_{2}\right)\left(q_{1}-1\right) H_{q_{1}-2}\left(X_{i_{1}}\right) H_{q_{2}-1}\left(X_{i_{2}}\right) H_{q_{3}-1}\left(X_{i_{3}}\right) \\
& \times \rho\left(i_{1}-i_{2}\right) \rho\left(i_{1}-i_{3}\right) .
\end{aligned}
$$

Therefore, by Fatou's lemma,

$$
\mathbb{E}\left(\left|D_{u_{n}}^{2} F_{n}\right|^{2}\right) \leq \liminf _{N \rightarrow \infty} \mathbb{E}\left(\left|\Phi_{n, N}^{2}\right|\right) .
$$

Then,

$$
\begin{aligned}
\left|\Phi_{n, N}\right|^{2} & =\frac{1}{n^{3}} \sum_{i_{1}, \ldots, i_{6}=1}^{n} \sum_{q_{1}, \ldots, q_{6}=2}^{N} C_{q} H_{q_{1}-2}\left(X_{i_{1}}\right) H_{q_{2}-1}\left(X_{i_{2}}\right) H_{q_{3}-1}\left(X_{i_{3}}\right) \\
& \times H_{q_{4}-2}\left(X_{i_{4}}\right) H_{q_{5}-1}\left(X_{i_{5}}\right) H_{q_{6}-1}\left(X_{i_{6}}\right) \rho\left(i_{1}-i_{2}\right) \rho\left(i_{1}-i_{3}\right) \rho\left(i_{4}-i_{5}\right) \rho\left(i_{4}-i_{6}\right),
\end{aligned}
$$

where

$$
C_{q}=c_{q_{1}} c_{q_{2}} c_{q_{3}} c_{q_{4}} c_{q_{5}} c_{q_{6}}\left(q_{1}+q_{2}\right)\left(q_{1}-1\right)\left(q_{4}+q_{5}\right)\left(q_{4}-1\right) .
$$

Using the product formula for multiple integrals (see Lemma 3.1), we get

$$
\begin{gathered}
\mathbb{E}\left(\left|\Phi_{n, N}\right|^{2}\right)=\frac{1}{n^{3}} \sum_{i_{1}, \ldots, i_{6}=1}^{n} \sum_{q_{1}, \ldots, q_{6}=2}^{N} \sum_{\beta \in \mathcal{D}_{q}} K_{q, \beta}\left(\prod_{1 \leq k<l \leq 6} \rho\left(i_{k}-i_{l}\right)^{\beta_{k l}}\right) \\
\times \rho\left(i_{1}-i_{2}\right) \rho\left(i_{1}-i_{3}\right) \rho\left(i_{4}-i_{5}\right) \rho\left(i_{4}-i_{6}\right),
\end{gathered}
$$

where

and

$$
K_{q, \beta}=\frac{\left(q_{1}+q_{2}\right)\left(q_{4}+q_{5}\right) \prod_{j=1}^{6} c_{q_{j}}\left(q_{j}-1\right) !}{\prod_{1 \leq k<l \leq 6} \beta_{k l} !}
$$

$\mathcal{D}_{q}=\left\{\left(\beta_{k l}\right)_{1 \leq k<l \leq 6}: \sum_{k \text { or } l=j} \beta_{k l}=q_{j}-1\right.$ for $j=2,3,5,6$ and $\sum_{k \text { or } l=j} \beta_{k l}=q_{j}-2$ for $\left.j=1,4\right\}$. 
Replacing $\beta_{j k}+1$ by $\beta_{j k}$ for $(j, k) \in\{(1,2),(1,3),(4,5),(4,6)\}$, yields

$$
\mathbb{E}\left(\left|\psi_{n, N}\right|^{2}\right)=\frac{1}{n^{3}} \sum_{i_{1}, \ldots, i_{6}=1}^{n} \sum_{q_{1}, \ldots, q_{6}=2}^{N} \sum_{\beta \in \mathcal{C}_{q}} L_{q, \beta}\left(\prod_{1 \leq k<l \leq 6} \rho\left(i_{k}-i_{l}\right)^{\beta_{k l}}\right),
$$

where

$$
L_{q, \beta}=\frac{\left(q_{1}+q_{2}\right)\left(q_{4}+q_{5}\right) \prod_{i=1}^{6} c_{q_{i}}\left(q_{i}-1\right) !}{\left(\beta_{12}+1\right) !\left(\beta_{13}+1\right) ! \beta_{14} ! \beta_{15} ! \beta_{16} ! \beta_{23} ! \beta_{24} ! \beta_{25} ! \beta_{26} ! \beta_{34} ! \beta_{35} ! \beta_{36} !\left(\beta_{45}+1\right) !\left(\beta_{46}+1\right) ! \beta_{56} !}
$$

and

$$
\mathcal{C}_{q}=\left\{\left(\beta_{k l}\right)_{1 \leq k<l \leq 6}: \sum_{k \text { or } l=j} \beta_{k l}=q_{j} \text { for } j=1, \ldots, 6 \text { and } \beta_{12}, \beta_{13}, \beta_{45} \cdot \beta_{46} \geq 1\right\} .
$$

Then, we can write

$$
\left.\left.\mathbb{E}\left(\mid \psi_{n, N}\right)\right|^{2}\right) \leq \sup _{\beta \in \mathcal{C}_{q}} A_{n, \beta} \sum_{q_{1}, \ldots, q_{6}=2}^{N} \sum_{\beta \in \mathcal{C}_{q}}\left|L_{q, \beta}\right|,
$$

where

$$
A_{n, \beta}=\frac{1}{n^{3}} \sum_{i_{1}, i_{2}, i_{3}, i_{4}=1}^{n} \prod_{1 \leq j<k \leq 6}\left|\rho\left(i_{i}-i_{k}\right)\right|^{\beta_{j k}}
$$

and the supremum is taken over all sets of nonnegative integers $\beta_{j k}, 1 \leq j<k \leq 6$, satisfying $\beta_{12}, \beta_{13}, \beta_{45}, \beta_{46} \geq 1, \beta_{j k} \leq 2$ for $1 \leq j<k \leq 6$ and

$$
2 \leq \sum_{j \text { or } k=\ell} \beta_{j k}, \quad \text { for } \quad 1 \leq \ell \leq 6
$$

Then, the estimation follows as in the proof of the last part of Theorem 1.2 ,

Now, we need to show that

$$
\sum_{q_{1}, \ldots, q_{6}=2}^{\infty} \sum_{\beta \in \mathcal{C}_{q}}\left|L_{q, \beta}\right|<\infty
$$

In fact,

$$
\begin{aligned}
& \sum_{q_{1}, \ldots, q_{6}=2}^{N} \sum_{\beta \in \mathcal{C}_{q}}\left|L_{q, \beta}\right|=\sum_{q_{1}, \ldots, q_{6}=2}^{N}\left(\prod_{i=1}^{6}\left|c_{q_{i}}\right|\right)\left(q_{1}+q_{2}\right)\left(q_{1}-1\right)\left(q_{3}+q_{4}\right)\left(q_{4}-1\right) \\
& \quad \times \mathbb{E}\left(H_{q_{1}-2}\left(X_{1}\right) H_{q_{2}-1}\left(X_{1}\right) H_{q_{3}-1}\left(X_{1}\right) H_{q_{4}-2}\left(X_{1}\right) H_{q_{5}-1}\left(X_{1}\right) H_{q_{6}-1}\left(X_{1}\right)\right) \\
& \left.=\mathbb{E}\left(A\left(g^{\prime \prime}\right)^{(N)}\right)^{2}\left(A\left(g_{1}\right)^{(N)}\right)^{4}\right) \leq\left\|A\left(g^{\prime \prime}\right)^{(N)}\right\|_{L^{6}(\mathbb{R}, \gamma)}^{\frac{1}{3}}\left\|A\left(g_{1}\right)^{(N)}\right\|_{L^{6}(\mathbb{R}, \gamma)}^{\frac{2}{3}}
\end{aligned}
$$

Since $A(g) \in \mathbb{D}^{3,6},\left(A\left(g^{\prime \prime}\right)^{(N)}\right)^{3}$ and $\left(A\left(g_{1}\right)^{(N)}\right)^{3}$ converge to $A\left(g^{\prime \prime}\right)$ and $A\left(g_{1}\right)$, respectively, in $L^{2}(\mathbb{R}, \gamma)$ by (3.1). Then, (6.8) is true. 
Step 4. We proceed to the estimation of $\sqrt{\mathbb{E}\left(\left|D_{u_{n}}^{3} F_{n}\right|^{2}\right)}$. Taking the derivative in (6.7), yields

$$
\begin{aligned}
D\left(D_{u_{n}}^{2} F_{n}\right) & =\frac{1}{n^{3 / 2}} \sum_{i, j, k=1}^{n} g^{\prime \prime \prime}\left(X_{i}\right) g_{1}\left(X_{j}\right) g_{1}\left(X_{k}\right) \rho(i-j) \rho(i-k) e_{i} \\
& +g^{\prime \prime}\left(X_{i}\right) g_{1}^{\prime}\left(X_{j}\right) g_{1}\left(X_{k}\right) \rho(i-j) \rho(i-k) e_{j}+g^{\prime \prime}\left(X_{i}\right) g_{1}\left(X_{j}\right) g_{1}^{\prime}\left(X_{k}\right) \rho(i-j) \rho(i-k) e_{k} \\
& +g^{\prime \prime}\left(X_{i}\right) g_{1}^{\prime}\left(X_{j}\right) g_{1}\left(X_{k}\right) \rho(i-j) \rho(j-k) e_{i}+g^{\prime}\left(X_{i}\right) g_{1}^{\prime \prime}\left(X_{j}\right) g_{1}\left(X_{k}\right) \rho(i-j) \rho(j-k) e_{j} \\
& +g^{\prime}\left(X_{i}\right) g_{1}^{\prime}\left(X_{j}\right) g_{1}^{\prime}\left(X_{k}\right) \rho(i-j) \rho(j-k) e_{k} .
\end{aligned}
$$

This implies

$$
\begin{aligned}
\left\langle u_{n}, D\left(D_{u_{n}}^{2} F_{n}\right\rangle_{\mathfrak{H}}=\right. & \frac{1}{n^{2}} \sum_{i_{1}, i_{2}, i_{3}, i_{4}=1}^{n} g^{\prime \prime \prime}\left(X_{i_{1}}\right) g_{1}\left(X_{i_{2}}\right) g_{1}\left(X_{i_{3}}\right) g_{1}\left(X_{i_{4}}\right) \rho\left(i_{1}-i_{2}\right) \rho\left(i_{1}-i_{3}\right) \rho\left(i_{1}-i_{4}\right) \\
& +g^{\prime \prime}\left(X_{i_{1}}\right) g_{1}^{\prime}\left(X_{i_{2}}\right) g_{1}\left(X_{i_{3}}\right) g_{1}\left(X_{i_{4}}\right) \rho\left(i_{1}-i_{2}\right) \rho\left(i_{1}-i_{3}\right) \rho\left(i_{2}-i_{4}\right) \\
& +g^{\prime \prime}\left(X_{i_{1}}\right) g_{1}\left(X_{i_{2}}\right) g_{1}^{\prime}\left(X_{i_{3}}\right) g_{1}\left(X_{i_{4}}\right) \rho\left(i_{1}-i_{2}\right) \rho\left(i_{1}-i_{3}\right) \rho\left(i_{3}-i_{4}\right) \\
& +g^{\prime \prime}\left(X_{i_{1}}\right) g_{1}^{\prime}\left(X_{i_{2}}\right) g_{1}\left(X_{i_{3}}\right) g_{1}\left(X_{i_{4}}\right) \rho\left(i_{1}-i_{2}\right) \rho\left(i_{2}-i_{3}\right) \rho\left(i_{1}-i_{4}\right) \\
& +g^{\prime}\left(X_{i_{1}}\right) g_{1}^{\prime \prime}\left(X_{i_{2}}\right) g_{1}\left(X_{i_{3}}\right) g_{1}\left(X_{i_{4}}\right) \rho\left(i_{1}-i_{2}\right) \rho\left(i_{2}-i_{3}\right) \rho\left(i_{2}-i_{4}\right) \\
& +g^{\prime}\left(X_{i_{1}}\right) g_{1}^{\prime}\left(X_{i_{2}}\right) g_{1}^{\prime}\left(X_{i_{3}}\right) g_{1}\left(X_{i_{4}}\right) \rho\left(i_{1}-i_{2}\right) \rho\left(i_{2}-i_{3}\right) \rho\left(i_{3}-i_{4}\right)
\end{aligned}
$$

Notice that the second, third and fourth terms are identical. This allows us to write

$$
\begin{aligned}
D_{u_{n}}^{3} F_{n}= & \frac{1}{n^{2}} \sum_{i_{1}, i_{2}, i_{3}, i_{4}=1}^{n} g^{\prime \prime \prime}\left(X_{i_{1}}\right) g_{1}\left(X_{i_{2}}\right) g_{1}\left(X_{i_{3}}\right) g_{1}\left(X_{i_{4}}\right) \rho\left(i_{1}-i_{2}\right) \rho\left(i_{1}-i_{3}\right) \rho\left(i_{1}-i_{4}\right) \\
& +3 g^{\prime \prime}\left(X_{i_{1}}\right) g_{1}^{\prime}\left(X_{i_{2}}\right) g_{1}\left(X_{i_{3}}\right) g_{1}\left(X_{i_{4}}\right) \rho\left(i_{1}-i_{2}\right) \rho\left(i_{1}-i_{3}\right) \rho\left(i_{2}-i_{4}\right) \\
& +g^{\prime}\left(X_{i_{1}}\right) g_{1}^{\prime \prime}\left(X_{i_{2}}\right) g_{1}\left(X_{i_{3}}\right) g_{1}\left(X_{i_{4}}\right) \rho\left(i_{1}-i_{2}\right) \rho\left(i_{2}-i_{3}\right) \rho\left(i_{2}-i_{4}\right) \\
& +g^{\prime}\left(X_{i_{1}}\right) g_{1}^{\prime}\left(X_{i_{2}}\right) g_{1}^{\prime}\left(X_{i_{3}}\right) g_{1}\left(X_{i_{4}}\right) \rho\left(i_{1}-i_{2}\right) \rho\left(i_{2}-i_{3}\right) \rho\left(i_{3}-i_{4}\right) .
\end{aligned}
$$

Then, we have

$$
D_{u_{n}}^{3} F_{n}=\lim _{N \rightarrow \infty} \Phi_{n, N}
$$

where the convergence holds in probability and

$$
\begin{aligned}
\Phi_{n, N} & =\frac{1}{n^{2}} \sum_{i_{1}, i_{2}, i_{3}, i_{4}=1}^{n} \sum_{q_{1}, q_{2}, q_{3}, q_{4}=2}^{N} C_{q}^{(1)} H_{q_{1}-3}\left(X_{i_{1}}\right) H_{q_{2}-1}\left(X_{i_{2}}\right) H_{q_{3}-1}\left(X_{i_{3}}\right) H_{q_{4}-1}\left(X_{i_{4}}\right) \\
& \times \rho\left(i_{1}-i_{2}\right) \rho\left(i_{1}-i_{3}\right) \rho\left(i_{1}-i_{4}\right) \\
& +C_{q}^{(2)} H_{q_{1}-2}\left(X_{i_{1}}\right) H_{q_{2}-2}\left(X_{i_{2}}\right) H_{q_{3}-1}\left(X_{i_{3}}\right) H_{q_{4}-1}\left(X_{i_{4}}\right) \rho\left(i_{1}-i_{2}\right) \rho\left(i_{1}-i_{3}\right) \rho\left(i_{2}-i_{4}\right) \\
& +C_{q}^{(3)} H_{q_{1}-1}\left(X_{i_{1}}\right) H_{q_{2}-3}\left(X_{i_{2}}\right) H_{q_{3}-1}\left(X_{i_{3}}\right) H_{q_{4}-1}\left(X_{i_{4}}\right) \rho\left(i_{1}-i_{2}\right) \rho\left(i_{2}-i_{3}\right) \rho\left(i_{2}-i_{4}\right) \\
& +C_{q}^{(4)} H_{q_{1}-1}\left(X_{i_{1}}\right) H_{q_{2}-2}\left(X_{i_{2}}\right) H_{q_{3}-2}\left(X_{i_{3}}\right) H_{q_{4}-1}\left(X_{i_{4}}\right) \rho\left(i_{1}-i_{2}\right) \rho\left(i_{2}-i_{3}\right) \rho\left(i_{1}-i_{4}\right)
\end{aligned}
$$


with

$$
\begin{aligned}
& C_{q}^{(1)}=c_{q_{1}} c_{q_{2}} c_{q_{3}} c_{q_{4}} q_{1}\left(q_{1}-1\right)\left(q_{1}-2\right), \\
& C_{q}^{(2)}=3 c_{q_{1}} c_{q_{2}} c_{q_{3}} c_{q_{4}} q_{1}\left(q_{1}-1\right)\left(q_{2}-1\right), \\
& C_{q}^{(3)}=c_{q_{1}} c_{q_{2}} c_{q_{3}} c_{q_{4}} q_{1}\left(q_{2}-1\right)\left(q_{2}-2\right), \\
& C_{q}^{(4)}=c_{q_{1}} c_{q_{2}} c_{q_{3}} c_{q_{4}} q_{1}\left(q_{2}-1\right)\left(q_{3}-1\right) .
\end{aligned}
$$

We can combine the first and third terms with the change of variables $\left(q_{1}, q_{2}\right) \rightarrow\left(q_{2}, q_{1}\right)$ and $\left(i_{1}, i_{2}\right) \rightarrow\left(i_{2}, i_{1}\right)$. In this way we obtain

$$
\begin{aligned}
\Phi_{n, N}= & \frac{1}{n^{2}} \sum_{i_{1}, i_{2}, i_{3}, i_{4}=1}^{n} \sum_{q_{1}, q_{2}, q_{3}, q_{4}=2}^{N} \widetilde{C}_{q}^{(1)} H_{q_{1}-3}\left(X_{i_{1}}\right) H_{q_{2}-1}\left(X_{i_{2}}\right) H_{q_{3}-1}\left(X_{i_{3}}\right) H_{q_{4}-1}\left(X_{i_{4}}\right) \\
& \times \rho\left(i_{1}-i_{2}\right) \rho\left(i_{1}-i_{3}\right) \rho\left(i_{1}-i_{4}\right) \\
& +\widetilde{C}_{q}^{(2)} H_{q_{1}-2}\left(X_{i_{1}}\right) H_{q_{2}-2}\left(X_{i_{2}}\right) H_{q_{3}-1}\left(X_{i_{3}}\right) H_{q_{4}-1}\left(X_{i_{4}}\right) \rho\left(i_{1}-i_{2}\right) \rho\left(i_{1}-i_{3}\right) \rho\left(i_{2}-i_{4}\right) \\
& +\widetilde{C}_{q}^{(3)} H_{q_{1}-1}\left(X_{i_{1}}\right) H_{q_{2}-2}\left(X_{i_{2}}\right) H_{q_{3}-2}\left(X_{i_{3}}\right) H_{q_{4}-1}\left(X_{i_{4}}\right) \rho\left(i_{1}-i_{2}\right) \rho\left(i_{2}-i_{3}\right) \rho\left(i_{1}-i_{4}\right) \\
= & : \Phi_{n, N}^{(1)}+\Phi_{n . N}^{(2)}+\Phi_{n . N}^{(3)}
\end{aligned}
$$

with

$$
\begin{aligned}
& \widetilde{C}_{q}^{(1)}=c_{q_{1}} c_{q_{2}} c_{q_{3}} c_{q_{4}}\left(q_{1}+q_{2}\right)\left(q_{1}-1\right)\left(q_{1}-2\right), \\
& \widetilde{C}_{q}^{(2)}=c_{q_{1}} c_{q_{2}} c_{q_{3}} c_{q_{4}} 3 q_{1}\left(q_{1}-1\right)\left(q_{2}-1\right), \\
& \widetilde{C}_{q}^{(3)}=c_{q_{1}} c_{q_{2}} c_{q_{3}} c_{q_{4}} q_{1}\left(q_{2}-1\right)\left(q_{3}-1\right) .
\end{aligned}
$$

Then, by Fatou's lemma,

$$
\mathbb{E}\left(\left|D_{u_{n}}^{3} F_{n}\right|^{2}\right) \leq \liminf _{N \rightarrow \infty} \mathbb{E}\left(\left|\Phi_{n, N}\right|^{2}\right) .
$$

We are going to treat each term $\Phi_{n, N}^{(i)}, i=1,2,3$, separately.

Case $i=1$. Let us first estimate $\mathbb{E}\left(\left|\Phi_{n, N}^{(1)}\right|^{2}\right)$. We have

$$
\begin{aligned}
\mathbb{E}\left(\left(\Phi_{n, N}^{(1)}\right)^{2}\right)= & \frac{1}{n^{4}} \sum_{i_{1}, \ldots, i_{8}=1}^{n} \sum_{q_{1}, \ldots, q_{8}=2}^{N} M_{q}^{(1)} \mathbb{E}\left(H_{q_{1}-3}\left(X_{i_{1}}\right) H_{q_{2}-1}\left(X_{i_{2}}\right) H_{q_{3}-1}\left(X_{i_{3}}\right) H_{q_{4}-1}\left(X_{i_{4}}\right)\right. \\
& \left.\times H_{q_{5}-3}\left(X_{i_{5}}\right) H_{q_{6}-1}\left(X_{i_{6}}\right) H_{q_{7}-1}\left(X_{i_{7}}\right) H_{q_{8}-1}\left(X_{i_{8}}\right)\right) \\
& \times \rho\left(i_{1}-i_{2}\right) \rho\left(i_{1}-i_{3}\right) \rho\left(i_{1}-i_{4}\right) \rho\left(i_{5}-i_{6}\right) \rho\left(i_{5}-i_{7}\right) \rho\left(i_{5}-i_{8}\right)
\end{aligned}
$$

where

$$
M_{q}^{(1)}=\left(\prod_{j=1}^{8} c_{q_{j}}\right)\left(q_{1}+q_{2}\right)\left(q_{1}-1\right)\left(q_{1}-2\right)\left(q_{5}+q_{6}\right)\left(q_{5}-1\right)\left(q_{5}-2\right) .
$$

This yields

$$
\begin{aligned}
\left.\mathbb{E}\left(\Phi_{n, N}^{(1)}\right)^{2}\right) \leq & \frac{1}{n^{4}} \sum_{i_{1}, \ldots, i_{8}=1}^{n} \sum_{q_{1}, \ldots q_{8}=2}^{N} \sum_{\beta \in \mathcal{D}_{q}^{(1)}} K_{q, \beta}^{(1)}\left(\prod_{1 \leq k<l \leq 8}\left|\rho\left(i_{k}-i_{l}\right)\right|^{\beta_{k l}}\right) \\
& \times\left|\rho\left(i_{1}-i_{2}\right) \rho\left(i_{1}-i_{3}\right) \rho\left(i_{1}-i_{4}\right) \rho\left(i_{5}-i_{6}\right) \rho\left(i_{5}-i_{7}\right) \rho\left(i_{5}-i_{8}\right)\right|,
\end{aligned}
$$


where

and

$$
K_{q, \beta}^{(1)}=\frac{\left(q_{1}+q_{2}\right)\left(q_{5}+q_{6}\right) \prod_{j=1}^{8}\left|c_{q_{j}}\right|\left(q_{j}-1\right) !}{\prod_{1 \leq k<l \leq 8} \beta_{k l} !},
$$

$$
\begin{gathered}
\mathcal{D}_{q}^{(1)}=\left\{\left(\beta_{k l}\right)_{1 \leq k<l \leq 8}: \sum_{k \text { or } l=j} \beta_{k l}=q_{j}-1 \text { for } j=2,3,4,6,7,8\right. \\
\text { and } \left.\sum_{k \text { or } l=j} \beta_{k l}=q_{j}-3 \text { for } j=1,5\right\} .
\end{gathered}
$$

Changing the exponents $\beta_{j k}+1$ in to $\beta_{j k}$ for $(j, k) \in\{(1,2),(1,3),(1,4),(5,6),(5,7),(5,8)\}$, we can write

$$
\mathbb{E}\left(\left(\Phi_{n, N}^{(1)}\right)^{2}\right) \leq \frac{1}{n^{4}} \sum_{i_{1}, \ldots, i_{8}=1}^{n} \sum_{q_{1}, \ldots q_{8}=2}^{N} \sum_{\beta \in \mathcal{C}_{q}^{(1)}} L_{q, \beta}^{(1)}\left(\prod_{1 \leq k<l \leq 8}\left|\rho\left(i_{k}-i_{l}\right)\right|^{\beta_{k l}}\right),
$$

where

$$
L_{q, \beta}^{(1)}=\frac{\left(q_{1}+q_{)}\left(q_{5}+q_{6}\right) \prod_{j=1}^{8}\left|c_{q_{j}}\right|\left(q_{j}-1\right) !\right.}{\left(\beta_{12}-1\right) !\left(\beta_{13}-1\right) !\left(\beta_{14}-1\right) !\left(\beta_{56}-1\right) !\left(\beta_{57}-1\right)\left(\beta_{58}-1\right) ! \prod_{(k, l) \in \mathcal{E}} \beta_{k l} !},
$$

with $\mathcal{E}=\{(k, l): 1 \leq k<l \leq 8,(k, l) \neq(1,2),(1,3),(1,4),(5,6),(5,7),(5,8)\}$ and

$$
\mathcal{C}_{q}^{(1)}=\left\{\left(\beta_{k l}\right)_{1 \leq k<l \leq 8}: \sum_{k \text { or } l=j} \beta_{k l}=q_{j} \text { for } j=1, \ldots, 8 \text { and } \beta_{12}, \beta_{13}, \beta_{14}, \beta_{56}, \beta_{57}, \beta_{58} \geq 1\right\} .
$$

Then, we obtain

where

$$
\mathbb{E}\left(\left(\Phi_{n, N}^{(1)}\right)^{2}\right) \leq \sup _{\beta \in \mathcal{C}_{q}^{(11)}} A_{n, \beta}^{(1)} \sum_{q_{1}, \ldots, q_{8}=2}^{N} \sum_{\beta \in \mathcal{C}_{q}^{(1)}}\left|L_{q, \beta}^{(1)}\right|
$$

$$
A_{n, \beta}^{(1)}=\frac{1}{n^{4}} \sum_{i_{1}, \ldots, i_{8}=1}^{n} \prod_{1 \leq j \leq k \leq 8}\left|\rho\left(i_{i}-i_{k}\right)\right|^{\beta_{j k}}
$$

and the supremum is taken over all sets of nonnegative integers $\beta_{j k}, 1 \leq j<k \leq 8$, satisfying $\beta_{12}, \beta_{13}, \beta_{14}, \beta_{56}, \beta_{57}, \beta_{58} \geq 1, \beta_{j k} \leq 2$ for $1 \leq j<k \leq 8$ and

$$
2 \leq \sum_{j \text { or } k=\ell} \beta_{j k}, \quad \text { for } \quad 1 \leq \ell \leq 8 .
$$

We need to estimate $A_{n, \beta}^{(1)}$ and to show that

$$
\sum_{q_{1}, \ldots, q_{8}=2}^{\infty} \sum_{\beta \in \mathcal{C}_{q}^{(1)}} L_{q, \beta}^{(1)}<\infty
$$

Estimation of $A_{n, \beta}^{(1)}$ : We claim that

$$
\sup _{\beta} A_{n, \beta}^{(1)} \leq C n^{-1}\left(\sum_{|k| \leq n}|\rho(k)|^{\frac{3}{2}}\right)^{4} .
$$


As in the proof of Theorem 1.2, we will make use of ideas from graph theory. The exponents $\beta_{j k}$ induce an unordered simple graph on the set of vertices $V=\{1,2,3,4,5,8\}$ by putting an edge between $j$ and $k$ whenever $\beta_{j k} \neq 0$. Because $\beta_{12}, \beta_{13} \geq 1, \beta_{14} \geq 1, \beta_{56} \geq 1, \beta_{57} \geq 1$ and $\beta_{58} \geq 1$, there are edges connecting the pairs of vertices $(1,2),(1,3),(1,4),(5,6),(5,7)$ and $(5,8)$. Condition (5.10) means that the degree of each vertex is at least 2 . Then we consider two cases, depending whether graph is connected or not.

Case 1: Suppose that the graph is not connected. This means that $\beta_{j k}=0$ if $j \in\{1,2,3,4\}$ and $k \in\{5,6,7,8\}$ and there is no edge between the sets $V_{1}=\{1,2,3,4\}$ and $V_{2}=\{5,6,7,8\}$. Therefore,

$$
A_{n, \beta}^{(1)} \leq\left(A_{n, \beta}^{(0)}\right)^{2}
$$

where

$$
A_{n, \beta}^{(0)}=\frac{1}{n^{2}} \sum_{i_{1}, \ldots, i_{4}=1}^{n} \prod_{1 \leq j \leq k \leq 4}\left|\rho\left(i_{i}-i_{k}\right)\right|^{\beta_{j k}}
$$

and the nonnegative integers $\beta_{j k}, 1 \leq j<k \leq 4$, satisfy $\beta_{12}, \beta_{13}, \beta_{14} \geq 1, \beta_{j k} \leq 2$ for $1 \leq j<k \leq 4$ and

$$
2 \leq \sum_{j \text { or } k=\ell} \beta_{j k}, \quad \text { for } \quad 1 \leq \ell \leq 4 .
$$

As a consequence, $\beta_{23}+\beta_{24} \geq 1, \beta_{23}+\beta_{34} \geq 1$ and $\beta_{24}+\beta_{34} \geq 1$. This means that at least two of the indices $\beta_{23}, \beta_{24}$ and $\beta_{34}$ is larger or equal to 1 . Considering the worst case, we can assume that $\beta_{23}=1$ and $\beta_{34}=1$. This leads to

$$
A_{n, \beta}^{(0)} \leq n^{-1} \sum_{\left|k_{1}\right|,\left|k_{2}\right|,\left|k_{3}\right| \leq n}\left|\rho\left(k_{1}\right) \rho\left(k_{2}\right) \rho\left(k_{3}\right) \rho\left(k_{2}-k_{1}\right) \rho\left(k_{3}-k_{2}\right)\right| .
$$

Using (2.11) and Hölder's inequality we obtain

$$
A_{n, \beta}^{(0)} \leq C n^{-1} \sum_{|k| \leq n}|\rho(k)| \leq C n^{-\frac{2}{3}}\left(\sum_{|k| \leq n}|\rho(k)|^{\frac{3}{2}}\right)^{\frac{2}{3}} .
$$

Case 2: Suppose that the graph is connected. This means that there is an edge connecting the sets $V_{1}$ and $V_{2}$. Suppose that $\beta_{\alpha_{0} \delta_{0}} \geq 1$, where $\alpha_{0} \in\{1,2,3,4\}$ and $\delta_{0} \in\{5,6,7,8\}$. We have then 7 nonzero coefficients $\beta$ : $\beta_{13}, \beta_{13}, \beta_{14}, \beta_{56}, \beta_{57}, \beta_{58}$ and $\beta_{\alpha_{0} \delta_{0}}$. Because all the edges have at least degree 2 , there must be another nonzero coefficient $\beta$. Assume it is $\beta_{\alpha_{1} \delta_{1}}$. Then, the worse case will be when $\beta_{12}=\beta_{13}=\beta_{14}=\beta_{56}=\beta_{57}=\beta_{58}=\beta_{\alpha_{0} \delta_{0}}=\beta_{\alpha_{1} \delta_{1}}=1$ and all the other coefficients are zero. Consider the change of variables $i_{1}-i_{2}=k_{1}, i_{1}-i_{3}=k_{2}$, $i_{1}-i_{4}=k_{3}, i_{5}-i_{6}=k_{4}, i_{5}-i_{7}=k_{5}, i_{5}-i_{8}=k_{6}, i_{\alpha_{0}}-i_{\delta_{0}}=k_{7}$. Then, it is easy to show that $i_{\alpha_{1}}-i_{\delta_{1}}=\mathbf{k} \cdot \mathbf{v}$, where $\mathbf{k}=\left(k_{1}, \ldots, k_{5}\right)$ and $\mathbf{v}$ is a 7 -dimensional vector whose components are 0,1 or -1 . Applying (2.10) and Hölder's inequality yields

$$
A_{n, \beta}^{(1)} \leq C n^{-2}\left(\sum_{|k| \leq n}|\rho(k)|\right)^{6} \leq C n^{-2}\left(\sum_{|k| \leq n}|\rho(k)|^{\frac{3}{2}}\right)^{4} .
$$

This completes the proof of (6.10). 
Proof of (6.9): We have

$$
\begin{aligned}
\sum_{q_{1}, \ldots, q_{8}=2}^{\infty} \sum_{\beta \in \mathcal{C}_{q}^{(1)}} L_{q, \beta}^{(1)}= & \mathbb{E}\left(\mid\left(A\left(g^{\prime \prime \prime}\right)^{(N)}\right)\left(X_{1}\right)\left(A\left(g_{1}\right)^{(N)}\left(X_{1}\right)\right)^{3}\right. \\
& \left.+\left.\left(A\left(g^{\prime}\right)^{(N)}\right)\left(X_{1}\right)\left(A\left(g^{\prime \prime}\right)^{(N)}\right)\left(X_{1}\right)\left(A\left(g_{1}\right)^{(N)}\left(X_{1}\right)\right)^{2}\right|^{2}\right) .
\end{aligned}
$$

Applying Hölder's inequality, yields

$$
\begin{aligned}
\sum_{q_{1}, \ldots, q_{8}=2}^{\infty} \sum_{\beta \in \mathcal{C}_{q}^{(1)}} L_{q, \beta}^{(1)} \leq & 2\left\|A\left(g^{\prime \prime \prime}\right)^{(N)}\right\|_{L^{8}(\mathbb{R}, \gamma)}^{2}\left\|A\left(g_{1}\right)^{(N)}\right\|_{L^{8}(\mathbb{R}, \gamma)}^{6} \\
& +2\left\|A\left(g^{\prime}\right)^{(N)}\right\|_{L^{8}(\mathbb{R}, \gamma)}^{2}\left\|A\left(g^{\prime \prime}\right)^{(N)}\right\|_{L^{8}(\mathbb{R}, \gamma)}^{2}\left\|A\left(g_{1}\right)^{(N)}\right\|_{L^{8}(\mathbb{R}, \gamma)}^{4} .
\end{aligned}
$$

By Proposition 3.2 and our hypothesis, taking the limit as $N$ tends to infinity, it follows that

$$
\begin{aligned}
\sum_{q_{1}, \ldots, q_{8}=2}^{\infty} \sum_{\beta \in \mathcal{C}_{q}^{(1)}} L_{q, \beta}^{(1)} \leq & 2\left\|A\left(g^{\prime \prime \prime}\right)\right\|_{L^{8}(\mathbb{R}, \gamma)}^{2}\left\|A\left(g_{1}\right)\right\|_{L^{8}(\mathbb{R}, \gamma)}^{6} \\
& +2\left\|A\left(g^{\prime}\right)\right\|_{L^{8}(\mathbb{R}, \gamma)}^{2}\left\|A\left(g^{\prime \prime}\right)\right\|_{L^{8}(\mathbb{R}, \gamma)}^{2}\left\|A\left(g_{1}\right)\right\|_{L^{8}(\mathbb{R}, \gamma)}^{4}<\infty .
\end{aligned}
$$

Case $i=2 . \quad$ For $\mathbb{E}\left[\left|\Phi_{n, N}^{(2)}\right|^{2}\right]$ we have

$$
\begin{aligned}
\mathbb{E}\left(\left(\Phi_{n, N}^{(2)}\right)^{2}\right)= & \frac{1}{n^{4}} \sum_{i_{1}, \ldots, i_{8}=1}^{n} \sum_{q_{1}, \ldots, q_{8}=2}^{N} M_{q}^{(2)} \mathbb{E}\left(H_{q_{1}-2}\left(X_{i_{1}}\right) H_{q_{2}-2}\left(X_{i_{2}}\right) H_{q_{3}-1}\left(X_{i_{3}}\right) H_{q_{4}-1}\left(X_{i_{4}}\right)\right. \\
& \left.\times H_{q_{5}-2}\left(X_{i_{5}}\right) H_{q_{6}-2}\left(X_{i_{6}}\right) H_{q_{7}-1}\left(X_{i_{7}}\right) H_{q_{8}-1}\left(X_{i_{8}}\right)\right) \\
& \times \rho\left(i_{1}-i_{2}\right) \rho\left(i_{1}-i_{3}\right) \rho\left(i_{2}-i_{4}\right) \rho\left(i_{5}-i_{6}\right) \rho\left(i_{5}-i_{7}\right) \rho\left(i_{6}-i_{8}\right)
\end{aligned}
$$

where

$$
\left.M_{q}^{(2)}=\left(\prod_{j=1}^{8} c_{q_{j}}\right) 9 q_{1}\left(q_{1}-1\right)\left(q_{2}-1\right) q_{5}\left(q_{5}-1\right)\left(q_{6}-1\right)\right) .
$$

This yields

$$
\begin{aligned}
\mathbb{E}\left(\left(\Phi_{n, N}^{(2)}\right)^{2}\right) \leq & \frac{1}{n^{4}} \sum_{i_{1}, \ldots, i_{8}=1}^{n} \sum_{q_{1}, \ldots q_{8}=2}^{N} \sum_{\beta \in \mathcal{D}_{q}^{(2)}} K_{q, \beta}^{(2)}\left(\prod_{1 \leq k<l \leq 8}\left|\rho\left(i_{k}-i_{l}\right)\right|^{\beta_{k l}}\right) \\
& \times\left|\rho\left(i_{1}-i_{2}\right) \rho\left(i_{1}-i_{3}\right) \rho\left(i_{2}-i_{4}\right) \rho\left(i_{5}-i_{6}\right) \rho\left(i_{5}-i_{7}\right) \rho\left(i_{6}-i_{8}\right)\right|,
\end{aligned}
$$

where

$$
K_{q, \beta}^{(2)}=\frac{9 q_{1} q_{5} \prod_{j=1}^{8}\left|c_{q_{j}}\right|\left(q_{j}-1\right) !}{\prod_{1 \leq k<l \leq 8} \beta_{k l} !}
$$

and

$$
\begin{gathered}
\mathcal{D}_{q}^{(2)}=\left\{\left(\beta_{k l}\right)_{1 \leq k<l \leq 8}: \sum_{k \text { or } l=j} \beta_{k l}=q_{j}-1 \text { for } j=3,4,7,8\right. \\
\left.\quad \text { and } \sum_{k \text { or } l=j} \beta_{k l}=q_{j}-2 \text { for } j=1,2,5,6\right\} .
\end{gathered}
$$


Changing the exponents $\beta_{j k}+1$ in to $\beta_{j k}$ for $(j, k) \in\{(1,2),(1,3),(2,4),(5,6),(5,7),(6,8)\}$, we can write

$$
\mathbb{E}\left(\left(\Phi_{n, N}^{(2)}\right)^{2}\right) \leq \frac{1}{n^{4}} \sum_{i_{1}, \ldots, i_{8}=1}^{n} \sum_{q_{1}, \ldots, q_{8}=2}^{N} \sum_{\beta \in \mathcal{C}_{q}^{(2)}} L_{q, \beta}^{(2)}\left(\prod_{1 \leq k<l \leq 8}\left|\rho\left(i_{k}-i_{l}\right)\right|^{\beta_{k l}}\right),
$$

where

$$
L_{q, \beta}^{(2)}=\frac{9 q_{1} q_{5} \prod_{j=1}^{8}\left|c_{q_{j}}\right|\left(q_{j}-1\right) !}{\left(\beta_{12}-1\right) !\left(\beta_{13}-1\right) !\left(\beta_{24}-1\right) !\left(\beta_{56}-1\right) !\left(\beta_{57}-1\right)\left(\beta_{68}-1\right) ! \prod_{(k, l) \in \mathcal{E}} \beta_{k l} !},
$$

with $\mathcal{E}=\{(k, l): 1 \leq k<l \leq 8,(k, l) \neq(1,2),(1,3),(2,4),(5,6),(5,7),(6,8)\}$ and

$$
\mathcal{C}_{q}^{(2)}=\left\{\left(\beta_{k l}\right)_{1 \leq k<l \leq 8}: \sum_{k \text { or } l=j} \beta_{k l}=q_{j} \text { for } j=1, \ldots, 8 \text { and } \beta_{12}, \beta_{13}, \beta_{24}, \beta_{56}, \beta_{57}, \beta_{6,8} \geq 1\right\} \text {. }
$$

Then, we have

$$
\left.\mathbb{E}\left(\Phi_{n, N}^{(2)}\right)^{2}\right) \leq \sup _{\beta \in \mathcal{C}_{q}^{(12)}} A_{n, \beta}^{(2)} \sum_{q_{1}, \ldots, q_{8}=2}^{N} \sum_{\beta \in \mathcal{C}_{q}^{(2)}}\left|L_{q, \beta}^{(2)}\right|
$$

where

$$
A_{n, \beta}^{(2)}=\frac{1}{n^{4}} \sum_{i_{1}, \ldots, i_{8}=1}^{n} \prod_{1 \leq j \leq k \leq 8}\left|\rho\left(i_{i}-i_{k}\right)\right|^{\beta_{j k}}
$$

and the supremum is taken over all sets of nonnegative integers $\beta_{j k}, 1 \leq j<k \leq 8$, satisfying $\beta_{12}, \beta_{13}, \beta_{24}, \beta_{56}, \beta_{57}, \beta_{68} \geq 1, \beta_{j k} \leq 2$ for $1 \leq j<k \leq 8$ and

$$
2 \leq \sum_{j \text { or } k=\ell} \beta_{j k}, \quad \text { for } \quad 1 \leq \ell \leq 8 .
$$

We need to estimate $A_{n, \beta}^{(2)}$ and to show that

$$
\sum_{q_{1}, \ldots, q_{8}=2}^{\infty} \sum_{\beta \in \mathcal{C}_{q}^{(2)}} L_{q, \beta}^{(2)}<\infty
$$

Estimation of $A_{n, \beta}^{(2)}$ : We claim that

$$
\sup _{\beta} A_{n, \beta}^{(2)} \leq C n^{-1}\left(\sum_{|k| \leq n}|\rho(k)|^{\frac{3}{2}}\right)^{4} .
$$

As in the proof of Theorem 1.2, we will make use of ideas from graph theory. The exponents $\beta_{j k}$ induce an unordered simple graph on the set of vertices $V=\{1,2,3,4,5,8\}$ by putting an edge between $j$ and $k$ whenever $\beta_{j k} \neq 0$. Because $\beta_{12} \geq 1, \beta_{13} \geq 1, \beta_{24} \geq 1, \beta_{56} \geq 1, \beta_{57} \geq 1$ and $\beta_{68} \geq 1$, there are edges connecting the pairs of vertices $(1,2),(1,3),(2,4),(5,6)(5,7)$ and $(6,8)$. Condition (5.10) means that the degree of each vertex is at least 2 . Then we consider two cases, depending whether graph is connected or not. 
Case 1: Suppose that the graph is not connected. This means that $\beta_{j k}=0$ if $j \in\{1,2,3,4\}$ and $k \in\{5,6,7,8\}$ and there is no edge between the sets $V_{1}=\{1,2,3,4\}$ and $V_{2}=\{5,6,7,8\}$. Therefore,

$$
A_{n, \beta}^{(2)} \leq\left(A_{n, \beta}^{(0)}\right)^{2}
$$

where

$$
A_{n, \beta}^{(0)}=\frac{1}{n^{2}} \sum_{i_{1}, \ldots, i_{4}=1}^{n} \prod_{1 \leq j \leq k \leq 4}\left|\rho\left(i_{i}-i_{k}\right)\right|^{\beta_{j k}}
$$

and the nonnegative integers $\beta_{j k}, 1 \leq j<k \leq 4$, satisfy $\beta_{12}, \beta_{13}, \beta_{24} \geq 1, \beta_{j k} \leq 2$ for $1 \leq j<k \leq 4$ and

$$
2 \leq \sum_{j \text { or } k=\ell} \beta_{j k}, \quad \text { for } \quad 1 \leq \ell \leq 4 .
$$

As a consequence, $\beta_{23}+\beta_{34} \geq 1$ and $\beta_{14}+\beta_{34} \geq 1$. This means $\beta_{34} \geq 1$ or both $\beta_{23}$ and $\beta_{14}$ are larger or equal than one. There are two possible cases:

(i) Suppose $\beta_{34} \geq 1$, Considering the worst case, we can assume that $\beta_{34}=1$. Then, applying (2.9) and Hölder's inequality, we obtain

$$
A_{n, \beta}^{(0)} \leq n^{-1} \sum_{\left|k_{1}\right|,\left|k_{2}\right|,\left|k_{3}\right| \leq n}\left|\rho\left(k_{1}\right) \rho\left(k_{2}\right) \rho\left(k_{3}\right) \rho\left(k_{1}+k_{3}-k_{2}\right)\right| \leq n^{-1}\left(\sum_{|k| \leq n}|\rho(k)|^{\frac{4}{3}}\right)^{3} .
$$

By Hölder's inequality, we can show that

$$
\left(A_{n, \beta}^{(0)}\right)^{2} \leq C n^{-1}\left(\sum_{|k| \leq n}|\rho(k)|^{\frac{3}{2}}\right)^{4} .
$$

(ii) Suppose $\beta_{23} \geq 1$ and $\beta_{14} \geq 1$. Then,

$$
A_{n, \beta}^{(0)} \leq n^{-1} \sum_{\left|k_{1}\right|,\left|k_{2}\right|,\left|k_{3}\right| \leq n}\left|\rho\left(k_{1}\right) \rho\left(k_{2}\right) \rho\left(k_{3}\right) \rho\left(k_{1}+k_{3}\right) \rho\left(k_{1}-k_{2}\right)\right|,
$$

and this case can be treated as (6.11).

Case 2: Suppose that the graph is connected. This means that there is an edge connecting the sets $V_{1}$ and $V_{2}$. Suppose that $\beta_{\alpha_{0} \delta_{0}} \geq 1$, where $\alpha_{0} \in\{1,2,3,4\}$ and $\delta_{0} \in\{5,6,7,8\}$. We have then 7 nonzero coefficients $\beta$ : $\beta_{12}, \beta_{13}, \beta_{24}, \beta_{56}, \beta_{57}, \beta_{68}$ and $\beta_{\alpha_{0} \delta_{0}}$. Because all the edges have at least degree 2 , there must be another nonzero coefficient $\beta$. Assume it is $\beta_{\alpha_{1} \delta_{1}}$. Then, the worse case will be when $\beta_{12}=\beta_{13}=\beta_{24}=\beta_{56}=\beta_{57}=\beta_{68}=\beta_{\alpha_{0} \delta_{0}}=\beta_{\alpha_{1} \delta_{1}}=1$ and all the other coefficients are zero. Consider the change of variables $i_{1}-i_{2}=k_{1}, i_{1}-i_{3}=k_{2}$, $i_{2}-i_{4}=k_{3}, i_{5}-i_{6}=k_{4}, i_{5}-i_{7}=k_{5}, i_{6}-i_{8}=k_{6}, i_{\alpha_{0}}-i_{\delta_{0}}=k_{7}$. Then, it is easy to show that $i_{\alpha_{1}}-i_{\delta_{1}}=\mathbf{k} \cdot \mathbf{v}$, where $\mathbf{k}=\left(k_{1}, \ldots, k_{5}\right)$ and $\mathbf{v}$ is a 7 -dimensional vector whose components are 0,1 or -1 . Then, using (2.10) and Hölder's inequality, we obtain

$$
A_{n, \beta}^{(1)} \leq C n^{-2}\left(\sum_{|k| \leq n}|\rho(k)|\right)^{6} \leq C n^{-2}\left(\sum_{|k| \leq n}|\rho(k)|^{\frac{3}{2}}\right)^{4} .
$$


Proof of (6.12): We have

$$
\begin{aligned}
\sum_{q_{1}, \ldots, q_{8}=2}^{\infty} \sum_{\beta \in \mathcal{C}_{q}^{(2)}} L_{q, \beta}^{(2)} & =9 \mathbb{E}\left(\left|A\left(g^{\prime \prime}\right)^{(N)}\left(X_{1}\right) A\left(g_{1}^{\prime}\right)\left(X_{1}\right) A\left(g_{1}\right)\left(X_{1}\right)^{2}\right|^{2}\right) \\
& \leq 9\left\|A\left(g^{\prime \prime}\right)^{(N)}\right\|_{L^{8}(\mathbb{R}, \gamma)}^{2}\left\|A\left(g_{1}^{\prime}\right)^{(N)}\right\|_{L^{8}(\mathbb{R}, \gamma)}^{2}\left\|A\left(g_{1}\right)^{(N)}\right\|_{L^{8}(\mathbb{R}, \gamma)}^{4},
\end{aligned}
$$

which converges as $N \rightarrow \infty$ to

$$
9\left\|A\left(g^{\prime \prime}\right)\right\|_{L^{8}(\mathbb{R}, \gamma)}^{2}\left\|A\left(g_{1}^{\prime}\right)\right\|_{L^{8}(\mathbb{R}, \gamma)}^{2}\left\|A\left(g_{1}\right)\right\|_{L^{8}(\mathbb{R}, \gamma)}^{4}<\infty .
$$

Case $i=3$. The term $\mathbb{E}\left[\left|\Phi_{n, N}^{(3)}\right|^{2}\right]$ can be handled in a similar way and we omit the details.

\section{REFERENCES}

[1] D. Bell and D. Nualart (2017). Noncentral limit theorem for the generalized Hermite process. Electron. J. Probab. 22,no. 2, 1-13.

[2] H. Biermé, A. Bonami, I. Nourdin and G. Peccati (2012). Optimal Berry-Esseen rates on the Wiener space: the barrier of third and fourth cumulants. ALEA, 9, no. 2, 473-500.

[3] H. J. Brascamp and E. H. Lieb (1976). Best constants in Youngs inequality, its converse, and its generalization to more than three functions. Adv. Math. 20, 151-173.

[4] P. Breuer and P. Major (1983). Central limit theorems for non-linear functionals of Gaussian fields. J. Mult. Anal. 13, 425-441.

[5] S. Campese, I. Nourdin and D. Nualart: Continuous Breuer-Major theorem: tightness and nonstationarity. Preprint.

[6] L. H. Y. Chen, L. Goldstein and Q.-M. Shao (2011). Normal approximation by Stein's method. SpringerVerlag, Berlin.

[7] L. Larsson-Cohn (2002). $L^{p}$-norms of Hermite polynomials and an extremal problem on Wiener chaos. Arkiv för Matematik 40, no. 1, 133-144.

[8] I. Nourdin and D. Nualart (2018). The functional Breuer-Major theorem. Preprint

[9] I. Nourdin and G. Peccati (2009). Stein's method on Wiener chaos. Probab. Theory Relat. Fields 145, no. $1,75-118$.

[10] I. Nourdin and G. Peccati (2012). Normal approximations with Malliavin calculus. From Stein's method to universality. Cambridge University Press.

[11] I. Nourdin and G. Peccati (2015). The optimal fourth moment theorem. Proc. Amer. Math.Soc. 143, 3123-3133.

[12] I. Nourdin, G. Peccati, X. Yang (2019). On rate of convergence for the Breuer-Major theorem. Preprint.

[13] D. Nualart (2006). The Malliavin calculus and related topics. Springer-Verlag, Berlin, second edition.

[14] D. Nualart (2009). Malliavin calculus and its applications. American Mathematical Society, CBMS regional conference series in mathematics.

[15] D. Nualart and E. Nualart (2018). Introduction fo Malliavin calculus. Cambridge University Press.

[16] D. Nualart and G. Peccati (2005). Central limit theorems for sequences of multiple stochastic integrals. Ann. Probab. 33, no. 1, 177-193.

[17] D. Nualart and H. Zhou (2018). Total variation estimates in the Breuer-Major theorem. Preprint

[18] G. Peccati and M. S. Taqqu (2011). Wiener chaos: moments, cumulants and diagrams - a survey with computer implementation. Springer-Verlag, Italia.

[19] C. Stein (1986). Approximate computation of expectations. Institute of Mathematical Statistics.

University of Kansas, Department of Mathematics, USA

E-mail address: sefika.kuzgun@ku.edu

University of Kansas, Department of Mathematics, USA

E-mail address: nualart@ku.edu 